\title{
Preferential attachment without vertex growth: emergence of the giant component
}

\author{
Svante Janson* and Lutz Warnke ${ }^{\dagger}$
}

April 25, 2019

\begin{abstract}
We study the following preferential attachment variant of the classical Erdös-Rényi random graph process. Starting with an empty graph on $n$ vertices, new edges are added one-by-one, and each time an edge is chosen with probability roughly proportional to the product of the current degrees of its endpoints (note that the vertex set is fixed). We determine the asymptotic size of the giant component in the supercritical phase, confirming a conjecture of Pittel from 2010. Our proof uses a simple method: we condition on the vertex degrees (of a multigraph variant), and use known results for the configuration model.
\end{abstract}

\section{Introduction}

During the last two decades 'dynamic' network models (which evolve/grow step-by-step) have been of great interest in various different research areas, including combinatorics, probability theory, statistical physics, and network science, see e.g. [5, 33, 17, 13, 19, 11, 39, 2]. Part of the motivation stems from the fact that many real-world networks (such as Facebook) also grow over time. Widely studied models include variants of the classical Erdős-Rényi random graph process [14, 5, 47, 48, 7] and the modern 'scale-free' preferential attachment model made popular by Barabási and Albert [3, 8, 2], which have strikingly different features.

In this paper we consider a hybrid between between the Erdős-Rényi and Barabási-Albert network models, where the vertex set is fixed (as in the Erdős-Rényi case) and edges are added with (one version of) preferential attachment. More precisely, let $\alpha \in(0, \infty)$ be a parameter, and write $\left(G_{n, m}^{\alpha}\right)_{m \geqslant 0}$ for the random graph process with fixed vertex set $[n]=\{1, \ldots, n\}$ where new edges are added one-by-one (starting with no edges) such that the next edge connects two currently non-adjacent vertices $v$ and $w$ with probability proportiona 1 to $\left(d_{v}+\alpha\right)\left(d_{w}+\alpha\right)$, where $d_{v}$ denotes the current degree of $v$. In intuitive words, edges are thus added according to a 'rich-get-richer' preferential attachment mechanism (since vertices with higher degree are more likely to be joined). Note that $G_{n, m}^{\alpha}$ has $m$ edges. Furthermore, in the limit $\alpha \rightarrow \infty$ all edges are added with the same probability, so we recover the Erdős-Rényi random graph process.

The dynamic network model $\left(G_{n, m}^{\alpha}\right)_{m \geqslant 0}$ is so natural that is has been suggested and studied multiple times (sometimes independently) in the complex networks and combinatorial probability literature. It was first studied in 2010 by Pittel [43], who described it is a special case of a more general model that he attributed to a suggestion by Lovász in 2002, where the next edge joins $v$ and $w$ with probability proportional to $f\left(d_{v}\right) f\left(d_{w}\right)$ for some function $f$; this in turn can be traced back to a suggestion 2 of Erdős and Rényi 15] from 1961 motivated by more realistic modeling (see also Section 5.2.2). From 2011 onwards Borgs, Chayes, Lovász, Sós, and Vesztergombi [9], Ráth and Szakács [45] and [30, Example 7.9] also studied a natural multigraph variant of $G_{n, m}^{\alpha}$ (see Section 2.1), motivated by the emerging (multi)graph limit theory paradigm. Furthermore,

\footnotetext{
*Department of Mathematics, Uppsala University, PO Box 480, SE-751 06 Uppsala, Sweden. E-mail: svante.janson@math.uu.se. Research partially supported by a grant from the Knut and Alice Wallenberg Foundation and a grant from the Simons foundation.

${ }^{\dagger}$ School of Mathematics, Georgia Institute of Technology, Atlanta GA 30332, USA. E-mail: warnke@math.gatech.edu. Research partially supported by NSF Grant DMS-1703516 and a Sloan Research Fellowship.

${ }^{1}$ Here we have tacitly normalized in a convenient way: namely, if the probability of adding the new edge $\{v, w\}$ is proportional to $\left(\chi d_{v}+\beta\right)\left(\chi d_{w}+\beta\right)$ with $\beta, \chi>0$, then it is also proportional to $\left(d_{v}+\alpha\right)\left(d_{w}+\alpha\right)$ with $\alpha:=\beta / \chi>0$.

${ }^{2}$ Erdős and Rényi proposed, even more generally, to study network models where the probability of joining $v$ and $w$ depends on the current degrees $d_{v}$ and $d_{w}$, see [15, p. 344].
} 
in 2012, Ben-Naim and Krapivsky [4] proposed and studied the model $G_{n, m}^{\alpha}$ by statistical physics methods, motivated by the way connections are formed on Facebook (see also [52] and Appendix A.2.2).

In this paper we study the emergence of the giant component in this intriguing model, which is one of the most important and fascinating phase transitions in random graph theory. Pittel [43] answered the basic question of existence and location of this phase transition in $G_{n, m}^{\alpha}$ : for any fixed $\alpha>0$ he showed that at around $m \approx m_{\mathrm{c}}$ many steps the largest component typically changes from size $\Theta(\log n)$ to size $\Theta(n)$, wher 3

$$
m_{\mathrm{c}}:=\frac{n}{2\left(1+\alpha^{-1}\right)}=\frac{n \alpha}{2(\alpha+1)} .
$$

A variant of this result for $\alpha=1$ was also reported by Ben-Naim and Krapivsky [4]. In fact, Pittel [43] proved much stronger estimates on the size $L_{1}(m)=L_{1}\left(G_{n, m}^{\alpha}\right)$ of the largest component of $G_{n, m}^{\alpha}$, in particular near the critical point $m_{\mathrm{c}}$. Focusing for simplicity on the 'supercritical' phase (where the unique 'giant' component has emerged as the largest component), his result [43, Theorem 1] can be written as follows 4] If $\varepsilon=O(1)$ and $\varepsilon^{4} n \rightarrow \infty$ as $n \rightarrow \infty$, then, for a certain function $\rho_{\alpha}(\varepsilon)$ with $\rho_{\alpha}(\varepsilon)=\Theta(\varepsilon)$ as $\varepsilon \searrow 0$, we have 5

$$
L_{1}\left(m_{\mathrm{c}}(1+\varepsilon)\right)=\rho_{\alpha}(\varepsilon) n \cdot\left(1+o_{p}(1)\right) .
$$

Overall, Pittel's 'finite-size scaling' results qualitatively recover several key features of the Erdős-Rényi phase transition [6, 7], in particular the fundamental 'linear growth' of the $\Theta(\varepsilon n)$-sized largest component, see (1.2). However, for technical reasons his proof requires the extra assumption $\varepsilon^{4} n \rightarrow \infty$, while it is natural to guess, and was conjectured by Pittel [43, pp. 621,649], that the estimate (1.2) remains valid under the weaker supercritical condition $\varepsilon^{3} n \rightarrow \infty$ known from the Erdős-Rényi reference model.

The main purpose of the present paper is to extend Pittel's result and verify his nice conjecture (which also appears in the recent book by Frieze and Karoński [17, Section 17.5]). We further extend the result by allowing $\alpha$ to depend on $n$. Moreover, and at least as important, we do this using a simpler method than the one in [43]: we use known results for the configuration model to derive the results rather quickly.

\section{$1.1 \quad$ Main results}

Our first result determines the asymptotic size of the giant component in the entire supercritical phase, thus confirming Pittel's nearly 10-year-old conjecture (see also Appendix A.2.1). Furthermore, (1.4) below identifies the precise linear growth-rate of the giant, generalizing and rigorizing a statistical physics result by Ben-Naim and Krapivsky [4] from 2012 for the special case $\alpha=1$ (see Appendix A.2.2).

Theorem 1.1 (Extending Pittel 43]). Fix $\alpha \in(0, \infty)$. If $\varepsilon=\varepsilon(n)=O(1)$ and $\varepsilon^{3} n \rightarrow \infty$ as $n \rightarrow \infty$, then

$$
L_{1}\left(m_{\mathrm{c}}(1+\varepsilon)\right)=\rho_{\alpha}(\varepsilon) n \cdot\left(1+o_{\mathrm{p}}(1)\right),
$$

where the continuous function $\rho_{\alpha}:(0, \infty) \rightarrow(0,1]$ is given by (4.13) together with (4.12); it satisfies $0<$ $\rho_{\alpha}(\varepsilon)<2 \varepsilon$ and

$$
\rho_{\alpha}(\varepsilon)=\frac{2 \varepsilon}{1+2 / \alpha}+O\left(\varepsilon^{2}\right), \quad \text { as } \varepsilon \searrow 0 .
$$

From the perspective of mathematical physics, this result places the preferential attachment process into the same universality class as the Erdős-Rényi reference model (with largest 'supercritical' component of order $\varepsilon n$ and largest 'subcritical' component of order $\varepsilon^{-2} \log \left(\varepsilon^{3} n\right)$, both under the condition $\varepsilon^{3} n \rightarrow \infty$, cf. [43]). This kind of universality is only known for relatively few network models, including random regular graphs [42], the configuration model [46], hypercube percolation [21, 24], and bounded-size Achlioptas processes [50].

Since the limiting case $\alpha \rightarrow \infty$ of the preferential attachment process recovers the uniform Erdős-Rényi process $\left(G_{n, m}^{\mathrm{ER}}\right)_{m \geqslant 0}$, it is natural to wonder under what conditions this Erdős-Rényi approximation holds rigorously in the case when $\alpha=\alpha(n)$ is finite but tends to infinity as $n \rightarrow \infty$. Our main giant component result, which is the following extension of Theorem 1.1. allows us to answer this intriguing question by allowing for $\alpha=\alpha(n)$, including $\alpha(n) \rightarrow \infty$. To establish uniqueness of the 'giant component' (as in [43]), Theorem 1.2 also includes a weak estimate on the size $L_{2}(m)=L_{2}\left(G_{n, m}^{\alpha}\right)$ of the second largest component of $G_{n, m}^{\alpha}$. We henceforth use the convention that $x / \infty=0$ for any finite $x$.

\footnotetext{
${ }^{3}$ The phase transition location $m_{\mathrm{c}}$ from 1.1 can easily be guessed via modern heuristics, see Appendix A.1

${ }^{4}$ See Appendix A.2.1 for Pittel's formulation of his supercritical giant component result.

${ }^{5}$ As usual, $o_{p}(1)$ denotes a quantity that converges to 0 in probability as $n \rightarrow \infty$; see e.g. [33, 29].
} 
Theorem 1.2 (Main giant component result). Assume that $\alpha=\alpha(n) \rightarrow a \in(0, \infty]$ as $n \rightarrow \infty$. If $\varepsilon=$ $\varepsilon(n)=O(1)$ and $\varepsilon^{3} n \rightarrow \infty$ as $n \rightarrow \infty$, then

$$
\begin{aligned}
& L_{1}\left(m_{\mathrm{c}}(1+\varepsilon)\right)=\rho_{a}(\varepsilon) n \cdot\left(1+o_{p}(1)\right), \\
& L_{2}\left(m_{\mathrm{c}}(1+\varepsilon)\right)=o_{p}(1) \cdot L_{1}\left(m_{\mathrm{c}}(1+\varepsilon)\right),
\end{aligned}
$$

where the function $\rho_{a}:(0, \infty) \rightarrow(0,1]$ is as in Theorem 1.1, and $\rho_{\infty}(\varepsilon)$ satisfies

$$
1-\rho_{\infty}(\varepsilon)=e^{-(1+\varepsilon) \rho_{\infty}(\varepsilon)} .
$$

In particular, (1.4) holds for any $\alpha \in(0, \infty]$.

Remark 4.1 also shows that $\lim _{\alpha \rightarrow \infty} \rho_{\alpha}(\varepsilon)=\rho_{\infty}(\varepsilon)$. Recognizing (1.7) as a standard branching process equation, the largest component of the Erdős-Rényi process $\left(G_{n, m}^{\mathrm{ER}}\right)_{m \geqslant 0}$ is well-known [6, 7] to satisfy

$$
L_{1}\left(G_{n, \frac{n}{2}(1+\varepsilon)}^{\mathrm{ER}}\right)=\rho_{\infty}(\varepsilon) n \cdot\left(1+o_{p}(1)\right)
$$

when $\varepsilon=O(1)$ and $\varepsilon^{3} n \rightarrow \infty$. From Theorem 1.2 it is easy to deduce (using continuity of $\rho_{\infty}$ ) that the preferential attachment process has the same giant component behaviour when $\alpha(n) \rightarrow \infty$ sufficiently fast.

Corollary 1.3 (Supercritical Erdős-Rényi behaviour). Assume that $\alpha=\alpha(n) \rightarrow \infty$ as $n \rightarrow \infty$. If $\varepsilon=$ $\varepsilon(n)=O(1), \varepsilon^{3} n \rightarrow \infty$ and $\alpha \varepsilon \rightarrow \infty$ as $n \rightarrow \infty$, then

$$
L_{1}\left(\frac{n}{2}(1+\varepsilon)\right)=L_{1}\left(G_{n, \frac{n}{2}(1+\varepsilon)}^{\alpha}\right)=\rho_{\infty}(\varepsilon) n \cdot\left(1+o_{\mathrm{p}}(1)\right) .
$$

In particular, $\varepsilon=O(1)$ and $\varepsilon^{3} n \rightarrow \infty$ imply (1.9) when $\alpha=\Omega\left(n^{1 / 3}\right)$.

For the interested reader we include two remarks about the function $\rho_{\alpha}$ and the condition $\varepsilon=O(1)$.

Remark 1.4. As shown in the proof in Section $4 \rho_{\alpha}(\varepsilon)$ is an analytic function of $\alpha \in(0, \infty)$ and $\varepsilon \in(0, \infty)$; moreover, it extends analytically to $\varepsilon \in[0, \infty)$, as shown in Remark 4.2 .

Remark 1.5. The condition $\varepsilon=O(1)$ in Theorems 1.1 1.2 can be removed. This is a trivial consequence of the monotonicity of the process and the fact that $\rho_{\alpha}(\varepsilon) \rightarrow 1$ as $\varepsilon \rightarrow \infty$, see Remark 4.1] it suffices to consider the case $\varepsilon \rightarrow \infty$, and then we may for any $\eta>0$ choose $\varepsilon_{0}$ such that $\rho_{a}\left(\varepsilon_{0}\right)>1-\eta$ and then whp (i.e., with probability tending to one as $n \rightarrow \infty$ ) we have

$$
n \geqslant L_{1}\left(m_{\mathrm{c}}(1+\varepsilon)\right) \geqslant L_{1}\left(m_{\mathrm{c}}\left(1+\varepsilon_{0}\right)\right) \geqslant\left(\rho_{a}\left(\varepsilon_{0}\right)-\eta\right) n \geqslant(1-2 \eta) n \geqslant(\rho(\varepsilon)-2 \eta) n,
$$

which also implies that $L_{2} \leqslant 2 \eta n$ whp.

\subsection{Comments}

To motivate why the phase transition of $G_{n, m}^{\alpha}$ has some Erdös-Rényi features (like linear growth of the giant component), let us point out that distinct tree-components $C_{1}, C_{2}$ merge with probability proportional to

$$
\sum_{v \in C_{1}}\left(d_{v}+\alpha\right) \cdot \sum_{w \in C_{2}}\left(d_{w}+\alpha\right)=\left[(2+\alpha)\left|C_{1}\right|-2\right] \cdot\left[(2+\alpha)\left|C_{2}\right|-2\right] .
$$

In concrete words, trees thus merge with rates that are approximately proportional to the rates $\left|C_{1}\right| \cdot\left|C_{2}\right|$ from the Erdős-Rényi process. Since the phase transition is usually dominated by the contribution of treelike components (e.g., by counting the vertices in 'small' trees outside of the giant component), after some hand-waving it thus becomes plausible to observe some key features of the Erdős-Rényi reference model.

Our actual proof takes a surprisingly simple different route (instead of trying to leverage the above treeheuristic). Indeed, we first show that a natural multigraph variant of $G_{n, m}^{\alpha}$ has, conditioned on its degree sequence, the same distribution as the well-known configuration model for random multigraphs. By noting that in the multigraph variant the degrees evolve nearly independently (when looked at in the right way), 
we can also get very strong control over the resulting asymptotic degree sequence of negative binomial form (which contrasts not only the Poisson distribution of the Erdős-Rényi model, but also the power-law distributions observed in many preferential attachment models). These two results together allow us to study the multigraph variant of $G_{n, m}^{\alpha}$ via standard results for the configuration model, which then easily gives the asymptotic size of the giant component in $G_{n, m}^{\alpha}$. See Section 2 for a detailed proof overview.

We mention that our arguments are quite different from Pittel [43], who studies the multigraph variant of $G_{n, m}^{\alpha}$ via involved enumerative techniques. In fact, he notes [43, p. 643] that by conditioning on the degree sequence, it might be possible to use known results for the configuration model; however, in the paper he used a different approach, partly because it seemed difficult to verify the required degree conditions. (We will see that this is not so difficult, using a continuous-time construction. Moreover, we have the advantage of being able to use a stream-lined version [32] of the original phase transition result [41] for the configuration model.)

Finally, while the focus of the present paper is on the giant component, it is important to note that our proof method can also be used to study other properties of $G_{n, m}^{\alpha}$ such as the $k$-core, see Section 5.3 .

\subsection{Organization}

In Section 2 we give a detailed overview of our proof strategy. In particular, we state several technical auxiliary results, which we later prove in Section 3. Furthermore, in Section 2.2 we give a heuristic argument of our main giant component result, which we make rigorous in Section 4 . In Section 5 we then discuss extensions, variants, other properties, and open problems. Finally, Appendix A.1 contains a simple heuristic for the phase transition location $m_{\mathrm{c}}$, and Appendix A.2 shows how our results are compatible with previous work.

\section{Proof structure}

In this section we outline our high-level proof strategy for Theorems 1.11.2, which proceeds roughly as follows. After introducing a suitable random multigraph variant $\left(G_{n, m}^{\alpha, *}\right)_{m \geqslant 0}$ of $\left(G_{n, m}^{\alpha}\right)_{m \geqslant 0}$, we shall derive three basic auxiliary results (whose proofs are deferred to Section 3). First, Theorem 2.2 shows that results for the random multigraph $G_{n, m}^{\alpha, *}$ transfer to the original random graph $G_{n, m}^{\alpha}$. Secondly, Theorem 2.4 shows that, by conditioning on its degree sequence, we can study $G_{n, m}^{\alpha, *}$ via the widely studied configuration model $G_{\mathbf{d}}^{*}$ for random multigraphs. Thirdly, Theorem 2.5 determines the typical degree sequence of $G_{n, m}^{\alpha, *}$. The crux is that these three results together allow us to study $G_{n, m}^{\alpha}$ by applying standard results for random graphs with given degree sequences (see Theorem 2.8), and in Section 2.2 we outline how this reduction makes our main giant component result plausible (the full details are deferred to Section 44).

\subsection{A multigraph variant: reduction and auxiliary results}

We start by introducing a convenient multigraph variant of $\left(G_{n, m}^{\alpha}\right)_{m \geqslant 0}$, which allows for loops and multiple edges (this natural model was also used by Pittel [43], and further studied from different aspects in [9, 45, 30]). We write $\left(G_{n, m}^{\alpha, *}\right)_{m \geqslant 0}$ for the random multigraph process with fixed vertex set $[n]$ and parameter $\alpha \in(0, \infty)$, where edges are added one-by-one (starting with no edges) such that the next edge connects distinct vertices $v$ and $w$ with probability proportional to $2\left(d_{v}+\alpha\right)\left(d_{w}+\alpha\right)$, and forms a loop at vertex $v$ with probability proportional to $\left(d_{v}+\alpha\right)\left(d_{v}+1+\alpha\right)$; here $d_{v}$ denotes the current degree of $v$ (as usual, each loop is counted as two edges at its endpoint). In this paper we shall first prove our main results for the random multigraph $G_{n, m}^{\alpha, *}$, which turns out to be much easier to analyze than $G_{n, m}^{\alpha}$; we record this intermediate goal for later reference.

Theorem 2.1 (Main multigraph result). Theorems 1.1 1.2 also hold for the random multigraph $G_{n, m}^{\alpha, *}$.

\subsubsection{Approximating $G_{n, m}^{\alpha}$ by the multigraph $G_{n, m}^{\alpha, *}$}

Our first auxiliary result allows us to study $G_{n, m}^{\alpha}$ via the random multigraph $G_{n, m}^{\alpha, *}$ (so that Theorems 1.11 .2 eventually follow from Theorem 2.1). In words, Theorem 2.2 implies that whp-results for $G_{n, m}^{\alpha, *}$ routinely transfer to $G_{n, m}^{\alpha}$ when $m=O(n)$ and $\alpha=\Omega(1)$. This lemma is basically the same as [43, Corollary 3]; one difference is that we do not restrict to constant $\alpha$. (Another difference is that we only give an $o(1)$ bound for the additive term in (2.1)-(2.2). This can easily be improved, but we do not need this.) 
Theorem 2.2 (Transfer statement: from $G_{n, m}^{\alpha, *}$ to $G_{n, m}^{\alpha}$, partly [43]). Given $C, \alpha_{0}>0$, there is $B=$ $B\left(C, \alpha_{0}\right)>0$ such that the following holds whenever $1 \leqslant m \leqslant C n$ and $\alpha \geqslant \alpha_{0}$. For any set $\mathcal{G}_{n}$ of graphs with $m$ edges and vertex set $[n]$, we have

$$
\mathbb{P}\left(G_{n, m}^{\alpha} \in \mathcal{G}_{n}\right) \leqslant B \cdot \mathbb{P}\left(G_{n, m}^{\alpha, *} \in \mathcal{G}_{n}\right)+o(1) .
$$

Remark 2.3. The proof shows more generally that, for any set $\mathcal{G}_{n, m}$ of graph sequences $\left(G_{0}, \ldots, G_{m}\right)$ with vertex set $[n]$, we have

$$
\mathbb{P}\left(\left(G_{n, i}^{\alpha}\right)_{0 \leqslant i \leqslant m} \in \mathcal{G}_{n, m}\right) \leqslant B \cdot \mathbb{P}\left(\left(G_{n, i}^{\alpha, *}\right)_{0 \leqslant i \leqslant m} \in \mathcal{G}_{n, m}\right)+o(1) .
$$

The proof strategy is to compare the stepwise conditional probabilities of the added edges, where we may clearly restrict to simple graph sequences (without loops or multiple edges). By construction, the conditional probability of adding the new edge $\{v, w\}$ is in both processes proportional to $2\left(d_{v}+\alpha\right)\left(d_{v}+\alpha\right)$, but the normalizing factors in the denominator differ slightly (since only one of these processes allows for loops and multiple edges; see (3.28)-(3.29) in Section 3.3). It turns out that during the first $m=O(n)$ steps the corresponding normalizing factors of both processes are extremely close together, which eventually allows us to establish (2.2), from which inequality (2.1) follows immediately. See Section 3.3 for the full details.

\subsubsection{Reducing the multigraph $G_{n, m}^{\alpha, *}$ to the configuration model}

Our second auxiliary result allows us to study $G_{n, m}^{\alpha, *}$ via the well-known configuration model $G_{\mathbf{d}}^{*}$ for random multigraphs, see e.g. [5, 17, 19]; it says that $G_{n, m}^{\alpha, *}$ conditioned on its degree sequence $d\left(G_{n, m}^{\alpha, *}\right)=\mathbf{d}=\left(d_{v}\right)_{v \in[n]}$ has the same distribution as the configuration model $G_{\mathbf{d}}^{*}=\left([n], E_{\mathbf{d}}\right)$, which we for concreteness here define as follows: Let $\mathcal{S}_{\mathbf{d}}$ be the set of all $2 m$-element sequences in which each vertex $v \in[n]$ appears $d_{v}$ times, pick a uniform random vertex sequence $\mathbf{w}=\left(w_{1}, \ldots, w_{2 m}\right) \in \mathcal{S}_{\mathbf{d}}$, and define the edge multiset $E_{\mathbf{d}}:=$ $\left\{w_{1} w_{2}, \ldots, w_{2 m-1} w_{2 m}\right\} 6$ As said above, this property was noted (but not exploited) by Pittel [43] (at least after conditioning the multigraph on being simple); it has also been noted or used in [45, 30].

Theorem 2.4 (Conditional equivalence: $G_{n, m}^{\alpha, *}$ and $G_{\mathbf{d}}^{*}$, 43, 45, 30]). For any $m \geqslant 1$ and any degree sequence $\mathbf{d}=\left(d_{v}\right)_{v \in[n]}$ with $\sum_{v \in[n]} d_{v}=2 m$, the random multigraph $G_{n, m}^{\alpha, *}$ conditioned on having degree sequence $\mathbf{d}$ has the same distribution as the configuration model $G_{\mathbf{d}}^{*}$. In other words, for any set $\mathcal{G}_{n}$ of multigraphs with $m$ edges and vertex set $[n]$, we have

$$
\mathbb{P}\left(G_{n, m}^{\alpha, *} \in \mathcal{G}_{n} \mid d\left(G_{n, m}^{\alpha, *}\right)=\mathbf{d}\right)=\mathbb{P}\left(G_{\mathbf{d}}^{*} \in \mathcal{G}_{n}\right) .
$$

As we shall see in Section 3.1, the random multigraph process was carefully designed to make (2.3) true 'by construction'. To make this plausible, recall that the conditional probability of adding the non-loop edge $\{v, w\}$ in the next step is proportional to $2 \cdot\left(d_{v}+\alpha\right) \cdot\left(d_{w}+\alpha\right)$. This 'factorization' suggests that we can alternatively construct the $m$ edges of $G_{n, m}^{\alpha, *}$ as follows: we first generate the vertex sequence $w_{1}, \ldots, w_{2 m}$ (each time vertex $v$ is chosen with probability proportional to $d_{v}+\alpha$ ) and then join them pairwise to the $m$ edges $w_{1} w_{2}, \ldots, w_{2 m-1} w_{2 m}$. In Section 3.1 we show that (a version of) this construction indeed gives the correct distribution (this is the place where the special treatment of loops is crucial). It furthermore turns out that every vertex sequence $\left(w_{1}, \ldots, w_{2 m}\right) \in \mathcal{S}_{\mathbf{d}}$ arises with the same probability (see (3.3) in Section 3.1), which by the described construction of $G_{\mathbf{d}}^{*}=\left([n], E_{\mathbf{d}}\right)$ then easily gives the desired conditional equivalence. See Section 3.1 for the full details.

\subsubsection{Approximating the degree sequence of $G_{n, m}^{\alpha, *}$}

Our third auxiliary result states that the degree sequence of $G_{n, m}^{\alpha, *}$ is asymptotically a negative binomial distribution $Y \sim \operatorname{NBin}(\alpha, p)$ with shape parameter $\alpha$ and suitable probability $p=p(\alpha, m / n)$, i.e.,

$$
\mathbb{P}(Y=r):=\left(\begin{array}{c}
\alpha+r-1 \\
r
\end{array}\right)(1-p)^{\alpha} p^{r}=\frac{\prod_{0 \leqslant j<r}(\alpha+j)}{r !}(1-p)^{\alpha} p^{r} \quad \text { for } r \in \mathbb{N}=\{0,1, \ldots\} .
$$

${ }^{6}$ This construction indeed gives the usual configuration model, since $E_{\mathbf{d}}$ has the same distribution as the edges of a uniform random matching of the $2 m$-element multiset in which each vertex $v \in[n]$ appears $d_{v}$ times. 
For notational convenience, given a degree sequence $\mathbf{d}=\left(d_{v}\right)_{v \in[n]}$ and an integer $k \geqslant 0$, we write

$$
\pi_{k}(\mathbf{d}):=\frac{1}{n} \sum_{v \in[n]} \mathbb{1}_{\left\{d_{v}=k\right\}} \quad \text { and } \quad \mu_{k}(\mathbf{d}):=\frac{1}{n} \sum_{v \in[n]} d_{v}^{k}
$$

for the proportion of vertices with degree $k$, and the $k$ th moment of the degree of a random vertex, respectively. We now state a limit theorem for the degrees (aiming at simplicity rather than the widest generality).

Theorem 2.5 (Degree sequence of $G_{n, m}^{\alpha, *}$ : negative binomial). Suppose that $n \rightarrow \infty, m=\Theta(n)$, and $\alpha=$ $\alpha(n)=\Omega(1)$. Let $\mathbf{d}$ be the (random) degree sequence of $G_{n, m}^{\alpha, *}$, and let $Y=Y_{n} \sim \operatorname{NBin}\left(\alpha, p_{n}\right)$ with

$$
p_{n}:=2 m /(n \alpha+2 m) .
$$

Then, for any sequence $\omega(n) \rightarrow \infty$, for every integer $k \geqslant 0$,

$$
\begin{aligned}
& \pi_{k}(\mathbf{d})=\mathbb{P}\left(Y_{n}=k\right)+o_{\mathrm{p}}\left(\omega(n) n^{-1 / 2}\right), \\
& \mu_{k}(\mathbf{d})=\mathbb{E} Y_{n}^{k}+o_{\mathrm{p}}\left(\omega(n) n^{-1 / 2}\right),
\end{aligned}
$$

where $\mathbb{E} Y_{n}^{k}=O(1)$.

Remark 2.6. The estimates (2.7)-(2.8) with an arbitrary $\omega(n) \rightarrow \infty$ can equivalently be written as $\pi_{k}(\mathbf{d})=$ $\mathbb{P}\left(Y_{n}=k\right)+O_{\mathrm{p}}\left(n^{-1 / 2}\right)$ and $\mu_{k}(\mathbf{d})=\mathbb{E} Y_{n}^{k}+O_{\mathrm{p}}\left(n^{-1 / 2}\right)$, see e.g. [29, Lemma 3].

Remark 2.7. We have $\mathbb{E} Y_{n}=2 m / n=\sum_{v \in[n]} d_{v} / n, \mathbb{E} Y_{n}\left(Y_{n}-1\right)=(2 m / n)^{2}\left(1+\alpha^{-1}\right)$, and $\mathbb{E} Y_{n}\left(Y_{n}-\right.$ 1) $\left(Y_{n}-2\right)=(2 m / n)^{3}\left(1+\alpha^{-1}\right)\left(1+2 \alpha^{-1}\right)$; see Lemma 3.1.

The proof strategy hinges on the fact that, in $G_{n, m}^{\alpha, *}$, the degree of a vertex equals the number of its concurrences in the auxiliary sequence $w_{1}, \ldots, w_{2 m}$ introduced above. To study these statistics we then switch to continuous time, and identify the degree of each vertex $v \in[n]$ with a suitable independent birth process (with initial value 0 and birth rates $\lambda_{k}:=k+\alpha$ ). In Section [3.2, we show that if we sequentially record the vertices which give birth, then the resulting vertex sequence $w_{1}, \ldots, w_{2 m}$ indeed has the correct distribution. This continuous-time embedding ensures (i) that the birth processes and thus the degrees evolve independently, and (ii) that each birth process has an explicit distribution at time $t$, which turns out to be of negative binomial form (see (3.6) in Section 3.2). These two properties make it easy to approximate the degree sequence up to the desired precision. See Section 3.2 for the full details.

\subsection{Reduction to configuration model $G_{\mathrm{d}}^{*}$ : giant component heuristics}

The punchline of the auxiliary results above is that we can obtain results for $G_{n, m}^{\alpha}$ by applying standard results for the well-understood configuration model $G_{\mathbf{d}}^{*}$, where $\mathbf{d}$ is random but approximates a negative binomial distribution. Armed with this reduction to the configuration model $G_{\mathbf{d}}^{*}$, the plan is to then estimate the size of the largest component by applying the following result of Janson and Luczak $\underline{32}$, a special case of Theorems 2.3-2.4], which is a convenient extension of the pioneering result by Molloy and Reed [41]. (The moment condition $\mu_{5}(\mathbf{d})=O(1)$ can be weakened to lower moments, see [32, 20], but we do not need this.)

Theorem 2.8 (Phase transition in $G_{\mathbf{d}}^{*}$, 32]). Suppose that, for each $n \geqslant n_{0}, \mathbf{d}=\left(d_{v}\right)_{v \in[n]}$ is a sequence of non-negative integers with $\mu_{5}(\mathbf{d})=O(1)$ such that $\sum_{v \in[n]} d_{v}$ is even. Furthermore, suppose that $D \in \mathbb{N}$ is a random variable that is independent of $n$ such that $\mathbb{E} D \in(0, \infty), \mathbb{P}(D=1)>0$, and $\pi_{k}(\mathbf{d}) \rightarrow \mathbb{P}(D=k)$ as $n \rightarrow \infty$, for every $k \geqslant 0$. Then, writing $L_{1}=L_{1}\left(G_{\mathbf{d}}^{*}\right)$ and $L_{2}=L_{2}\left(G_{\mathbf{d}}^{*}\right)$ for the sizes of the largest and second largest component of $G_{\mathbf{d}}^{*}$, the following holds.

(i) If $\mathbb{E} D(D-2)>0$, then there is a unique $\xi \in(0,1)$ satisfying $\mathbb{E} D \xi^{D}=\xi^{2} \mathbb{E} D$, and, furthermore,

$$
L_{1} / n \stackrel{\mathrm{p}}{\longrightarrow} 1-\mathbb{E} \xi^{D}>0 \quad \text { and } \quad L_{2} / n \stackrel{\mathrm{p}}{\longrightarrow} 0 .
$$

(ii) If $\mathbb{E} D(D-2)=0$ and $\zeta_{n}:=\sum_{v \in[n]} d_{v}\left(d_{v}-2\right)$ satisfies $\zeta_{n}>0$ and $n^{-2 / 3} \zeta_{n} \rightarrow \infty$ as $n \rightarrow \infty$, then

$$
L_{1}=\left(\frac{2 \mathbb{E} D}{\mathbb{E} D(D-1)(D-2)}+o_{p}(1)\right) \zeta_{n} \quad \text { and } \quad L_{2}=o_{p}\left(\zeta_{n}\right) .
$$


(iii) If $\mathbb{E} D(D-2) \leqslant 0$, then $L_{1} / n \stackrel{\mathrm{p}}{\longrightarrow} 0$.

In the following heuristic discussion we shall make our giant component results for $G_{n, m}^{\alpha}$ plausible (with a focus on Theorem 1.11). To this end, as discussed above, it suffices to study the giant component of $G_{\mathbf{d}}^{*}$ for 'typical' degree sequences d, where we shall below (for simplicity) assume that we can apply Theorem 2.8 with the random variable $D$ being approximately equal to $Y=Y_{n} \sim \operatorname{NBin}(\alpha, p)$ from Theorem 2.5, Regarding the point when the giant component emerges, in the usual informal language Theorem 2.8 (together with Remark 2.7] states that there is a giant component if and only if

$$
\mathbb{E} D(D-2) \approx \mathbb{E} Y(Y-2)=\mathbb{E} Y(Y-1)-\mathbb{E} Y=\frac{2 m}{n}\left(\frac{2 m}{n}\left(1+\alpha^{-1}\right)-1\right)
$$

is larger than zero, which makes the phase transition location $m_{\mathrm{c}} \approx n /\left[2\left(1+\alpha^{-1}\right)\right]$ plausible (see Appendix A.1 for an alternative heuristic). Regarding the size of the largest component for $m=m_{\mathrm{c}}(1+\varepsilon)$ with $\varepsilon=\varepsilon(n) \rightarrow 0$, in view of (2.11) we have $\mathbb{E} D(D-2) \approx \mathbb{E} Y(Y-2)=\Theta(\varepsilon) \rightarrow 0$. In this case Theorem 2.4(ii) intuitively predicts (together with (2.8) and Remark 2.7) that the size of the largest component is approximately

$$
\frac{2 \mathbb{E} D}{\mathbb{E} D(D-1)(D-2)} \cdot \sum_{v \in[n]} d_{v}\left(d_{v}-2\right) \approx \frac{2 \mathbb{E} Y \mathbb{E} Y(Y-2) n}{\mathbb{E} Y(Y-1)(Y-2)} \approx \frac{2 \varepsilon n}{1+2 \alpha^{-1}},
$$

which makes $L_{1}\left(m_{\mathrm{c}}(1+\varepsilon)\right) \approx \rho_{\alpha}(\varepsilon) n$ with $\rho_{\alpha}(\varepsilon) \approx 2 \varepsilon /\left(1+2 \alpha^{-1}\right)$ as $\varepsilon \searrow 0$ plausible. In fact, our above application of Theorem 2.q[(ii) tacitly required that the parameter

$$
n^{-2 / 3} \cdot \sum_{v \in[n]} d_{v}\left(d_{v}-2\right) \approx n^{1 / 3} \cdot \mathbb{E} Y(Y-2)=\Theta\left(n^{1 / 3} \varepsilon\right)
$$

tends to infinity, which makes the assumption $\varepsilon^{3} n \rightarrow \infty$ and thus Theorem 1.1 plausible. See Section 4 for a rigorous version of the above heuristic arguments (in the more general setting of Theorem 1.2).

\section{Proofs of auxiliary results}

In this section we prove the three basic auxiliary results stated in Sections 2.1.12.1.3 As noted above, the results have partly been shown earlier, but for completeness we give complete proofs of the versions used here. We consider Theorem 2.2 last, since we find it convenient to use Theorem 2.5 in the proof.

\subsection{Proof of Theorem 2.4: conditional equivalence of $G_{n, m}^{\alpha, *}$ and $G_{\mathbf{d}}^{*}$}

The conditional equivalence result of Theorem 2.4 can be shown in several ways, including enumeration [43] and exchangeability [45, 30] approaches. Inspired by Pólya urn arguments, here we shall use an elementary approach that avoids cumbersome explicit calculations by defining appropriate random variables (which also facilitates the upcoming degree sequence arguments). To this end we introduce a random sequence

$$
\left(W_{j}\right)_{j \geqslant 1}
$$

of vertices from $[n]$, by defining $W_{i+1}$ to have the conditional probability distribution with

$$
\mathbb{P}\left(W_{i+1}=v \mid W_{1}, \ldots, W_{i}\right)=\frac{\sum_{j \in[i]} \mathbb{1}_{\left\{W_{j}=w\right\}}+\alpha}{i+\alpha n} \quad \text { for all } v \in[n] .
$$

(This can be interpreted as the sequence of draws from a Pólya urn with $n$ colours, with initially $\alpha$ balls of each colour, see e.g. [40, 44, 35].) By joining these vertices pairwise to edges, for each $i \geqslant 0$ we then obtain a multigraph with vertex set $[n]$ and edge multiset $E_{n, i}:=\left\{W_{1} W_{2}, \ldots, W_{2 i-1} W_{2 i}\right\}$, where $E_{n, 0}=\varnothing$. The resulting multigraph sequence is easily seen to have the same distribution as $\left(G_{n, i}^{\alpha, *}\right)_{i \geqslant 0}$. Indeed, since $\sum_{j \in[2 i]} \mathbb{1}_{\left\{W_{j}=v\right\}}=d_{v}(i)$ equals the degree of vertex $v$ after $i$ steps, this follows from the simple observation that the stepwise conditional distributions of the added edges are the same (the conditional probability 
of adding $\{v, w\}$ as the next edge is proportional to $2 \cdot\left(d_{v}(i)+\alpha\right)\left(d_{w}(i)+\alpha\right)$ when $v \neq w$, and proportional to $\left(d_{v}(i)+\alpha\right)\left(d_{v}(i)+1+\alpha\right)$ when $\left.v=w\right)$. Now the proof of the desired conditional equivalence result is straightforward, since the above 'pairwise joining of $2 m$ vertices' construction of the edge multiset is similar to the construction of the configuration model $G_{\mathbf{d}}^{*}$ described in Section 2.1.2.

Proof of Theorem 2.4. Let $\mathbf{d}=\left(d_{v}\right)_{v \in[n]}$ be a degree sequence with $\sum_{v \in[n]} d_{v}=2 m$. Recalling that $\mathcal{S}_{\mathbf{d}}$ denotes the set of all $2 m$-element sequences $\mathbf{w}=\left(w_{1}, \ldots, w_{2 m}\right)$ in which each vertex $v \in[n]$ appears $d_{v}$ times, below we shall write $E(\mathbf{w}):=\left\{w_{1} w_{2}, \ldots, w_{2 m-1} w_{2 m}\right\}$ for the associated edge multiset. For any multigraph $G$ with degree sequence $d(G)=\mathbf{d}$, by the above-discussed construction of $G_{n, m}^{\alpha, *}$ it follows that

$$
\mathbb{P}\left(G_{n, m}^{\alpha, *}=G \mid d\left(G_{n, m}^{\alpha, *}\right)=\mathbf{d}\right)=\frac{\mathbb{P}\left(G_{n, m}^{\alpha, *}=G\right)}{\mathbb{P}\left(d\left(G_{n, m}^{\alpha, *}\right)=\mathbf{d}\right)}=\frac{\sum_{\mathbf{w} \in \mathcal{S}_{\mathbf{d}}: E(\mathbf{w})=E(G)} \mathbb{P}\left(\left(W_{1}, \ldots, W_{2 m}\right)=\mathbf{w}\right)}{\sum_{\mathbf{w} \in \mathcal{S}_{\mathbf{d}}} \mathbb{P}\left(\left(W_{1}, \ldots, W_{2 m}\right)=\mathbf{w}\right)} .
$$

By multiplying the conditional probabilities from (3.2) (and rearranging the factors in the numerator), it now is straightforward to see that the above probabilities

$$
\mathbb{P}\left(\left(W_{1}, \ldots, W_{2 m}\right)=\mathbf{w}\right)=\frac{\prod_{v \in[n]} \prod_{0 \leqslant j<d_{v}}(j+\alpha)}{\prod_{0 \leqslant i<2 m}(i+\alpha n)}
$$

are the same for all $\mathbf{w} \in \mathcal{S}_{\mathbf{d}}$ (as they depend on the degree sequence $\mathbf{d}=\left(d_{v}\right)_{v \in[n]}$ only). Since the edge multiset of $G_{\mathbf{d}}^{*}$ is defined as $E(\mathbf{w})$ for a uniform random $\mathbf{w} \in \mathcal{S}_{\mathbf{d}}$ (see Section 2.1.2), it readily follows that

$$
\mathbb{P}\left(G_{n, m}^{\alpha, *}=G \mid d\left(G_{n, m}^{\alpha, *}\right)=\mathbf{d}\right)=\sum_{\mathbf{w} \in \mathcal{S}_{\mathbf{d}}} \frac{\mathbb{1}_{\{E(\mathbf{w})=E(G)\}}}{\left|\mathcal{S}_{\mathbf{d}}\right|}=\mathbb{P}\left(G_{\mathbf{d}}^{*}=G\right),
$$

which implies the claimed conditional equivalence (since $G$ with degree sequence $d(G)=\mathbf{d}$ was arbitrary).

\subsection{Proof of Theorem 2.5; negative binomial degree sequence of $G_{n, m}^{\alpha, *}$}

For the degree sequence result of Theorem 2.5 it will be convenient to consider a continuous time embedding of the $\left(W_{j}\right)_{j \geqslant 1}$ based construction of $\left(G_{n, i}^{\alpha, *}\right)_{i} \geqslant 0$, since this will give us more independence. (This is a special case of a general embedding for Pólya urns, see e.g. [1, Section 9.2] with extension in [25, Remark 4.2] and [26, Remark 1.11].) To this end, let

$$
\left(\left(D_{v}(t)\right)_{t \in[0, \infty)}\right)_{v \in[n]}
$$

be independent pure birth processes with initial value 0 and birth rates $\lambda_{k}:=k+\alpha$ (i.e., the transition rate from state $k$ to state $k+1$ ). Identifying vertex $v \in[n]$ with the birth process $D_{v}(t)$, the order of the random birth-times $\left(\tau_{j}\right)_{j} \geqslant 1$ (also defining $\tau_{0}:=0$ for convenience) thus naturally induces a sequence of random vertices $\left(W_{j}\right)_{j \geqslant 1}$ (the ones which gave birth at the corresponding times). Justifying our slight abuse of notation, it is not difficult to check that this vertex sequence has the same distribution as the sequence $\left(W_{j}\right)_{j} \geqslant 1$ defined in Section 3.1 Indeed, since vertex $v$ occurs $\sum_{j \in[i]} \mathbb{1}_{\left\{W_{j}=v\right\}}=D_{v}\left(\tau_{i}\right)$ many times in $\left(W_{1}, \ldots, W_{i}\right)$, this follows from the simple observation that the stepwise conditional distributions of the selected vertices are the same (the conditional probability of selecting $v$ as the next vertex is $\left(D_{v}\left(\tau_{i}\right)+\alpha\right) /(i+\alpha n)$, since the total rate equals $\left.\sum_{w \in[n]}\left(D_{w}\left(\tau_{i}\right)+\alpha\right)=i+\alpha n\right)$. For later reference we also record the standard fact7 that $D_{v}(t)$ has a negative binomial distribution with shape parameter $\alpha$ and probability $1-e^{-t}$, i.e.,

$$
D_{v}(t) \sim \operatorname{NBin}\left(\alpha, 1-e^{-t}\right) .
$$

Exploiting independence of the birth-processes, now the proof of the desired degree sequence result is conceptually straightforward, since in our vertex-based construction of $G_{n, m}^{\alpha, *}$ the degree of vertex $v$ equals

$$
d_{v}(m)=\sum_{j \in[2 m]} \mathbb{1}_{\left\{W_{j}=v\right\}}=D_{v}\left(\tau_{2 m}\right)
$$

\footnotetext{
${ }^{7}$ By standard textbook results (see, e.g., $\left[18\right.$, Section 6.8]) the functions $p_{k}(t):=\mathbb{P}\left(D_{v}(t)=k\right.$ ) with $p_{k}(t)=\mathbb{1}_{\{k=0\}}$ are the unique solutions of the forward equations $p_{k}^{\prime}(t)=\lambda_{k-1} p_{k-1}(t) \mathbb{1}_{\{k \geqslant 1\}}-\lambda_{k} p_{k}(t)$. For $\lambda_{k}=k+\alpha$ the solution of these differential equations turns out to be $p_{k}(t)=\mathbb{P}(Y=k)$ with $Y \sim \operatorname{NBin}\left(\alpha, 1-e^{-t}\right)$ as defined in 2.4). This can alternatively be deduced from [18, Exercise 6.8.6], and is also explicitly stated in [55, (3.15)], for example.
} 
where $\tau_{2 m}$ will be highly concentrated. Before giving the details, we record some basic properties of negative binomial random variables (deferring their proofs, which are rather tangential to the main argument here). For $x \in \mathbb{R}$ and integer $k \geqslant 0$, we denote the falling factorial by $\langle x\rangle_{k}:=\prod_{0 \leqslant j<k}(x-j)$, where $\langle x\rangle_{0}=1$.

Lemma 3.1. For $Y \sim \operatorname{NBin}(\alpha, p)$ the following holds for all $\alpha \in(0, \infty)$ and $p \in[0,1)$.

(i) We have, for every integer $k \geqslant 0$,

$$
\mathbb{E}\langle Y\rangle_{k}=(\mathbb{E} Y)^{k} \cdot \prod_{1 \leqslant j<k}(1+j / \alpha) \quad \text { with } \quad \mathbb{E} Y=\frac{\alpha p}{1-p} .
$$

(ii) The probability generating function and its derivative are given by, for $|x|<1 / p$,

$$
\mathbb{E} x^{Y}=\left(\frac{1-p}{1-p x}\right)^{\alpha} \quad \text { and } \quad \mathbb{E}\left[Y x^{Y-1}\right]=\alpha p \frac{(1-p)^{\alpha}}{(1-p x)^{\alpha+1}} .
$$

(iii) If $p=1-e^{-t}$, then $t=\log (1+x / \alpha)$ implies $p=1-1 /(1+x / \alpha)=x /(x+\alpha)$ and $\mathbb{E} Y=x$.

Proof of Theorem 2.5. By the construction of $G_{n, m}^{\alpha, *}$ discussed above, we have, see (3.7),

$$
\mathbf{d}=d\left(G_{n, m}^{\alpha, *}\right)=\left(D_{v}\left(\tau_{2 m}\right)\right)_{v \in[n]} .
$$

With an eye on (2.5), we now introduce the auxiliary variables

$$
N_{k}(t):=\sum_{v \in[n]} \mathbb{1}_{\left\{D_{v}(t)=k\right\}} \quad \text { and } \quad M_{k}(t):=\sum_{v \in[n]} D_{v}(t)^{k} .
$$

We henceforth write $\omega=\omega(n)$, and may (as usual) assume that $\sqrt{\omega n} \leqslant 2 m$ holds. With the goal of approximating $\tau_{2 m}$ by a deterministic auxiliary time $t_{m}$, define

$$
t_{m}:=\log \left(1+\frac{2 m}{\alpha n}\right) \quad \text { and } \quad t_{m}^{ \pm}:=\log \left(1+\frac{2 m \pm \sqrt{\omega n}}{\alpha n}\right) .
$$

These times are chosen such that, by (3.6) and Lemma 3.1][(iii)] we have

$$
\begin{aligned}
D_{v}\left(t_{m}\right) & \stackrel{\mathrm{d}}{=} Y_{n} \sim \operatorname{NBin}\left(\alpha, p_{n}\right), \\
\mathbb{E} D_{v}\left(t_{m}^{ \pm}\right) & =\frac{2 m \pm \sqrt{\omega n}}{n}=\mathbb{E} D_{v}\left(t_{m}\right) \pm \sqrt{\omega / n}
\end{aligned}
$$

Furthermore, since we assume $\sqrt{\omega n} \leqslant 2 m$ and $m=O(n)$, all terms on the right-hand side of (3.14) are $O(1)$, so (3.8) and $\alpha=\Omega(1)$ yield, for every fixed integer $q \geqslant 0$,

$$
\max _{t \in\left\{t_{m}^{-}, t_{m}, t_{m}^{+}\right\}} \mathbb{E}\left\langle D_{v}(t)\right\rangle_{q}=O(1) .
$$

For every $k \geqslant 1$ it is well-known that we may write $x^{k}$ as a linear combination

$$
x^{k}=\sum_{1 \leqslant q \leqslant k}\left\{\begin{array}{l}
k \\
q
\end{array}\right\}\langle x\rangle_{q},
$$

where the coefficients are the so-called Stirling numbers of the second kind. Hence (3.15) also holds with $\left\langle D_{v}(t)\right\rangle_{q}$ replaced by $D_{v}(t)^{q}$, so that $\mathbb{E} Y_{n}^{q}=O(1)$ by (3.13). Using independence of the birth processes, we also infer

$$
\operatorname{Var} M_{k}\left(t_{m}^{ \pm}\right)=\sum_{v \in[n]} \operatorname{Var}\left[D_{v}\left(t_{m}^{ \pm}\right)^{k}\right] \leqslant \sum_{v \in[n]} \mathbb{E} D_{v}\left(t_{m}^{ \pm}\right)^{2 k}=O(n) .
$$

We now approximate $\tau_{2 m}$. To this end we consider $M_{1}(t)=\sum_{v \in[n]} D_{v}(t)$, where (3.14) yields

$$
\mathbb{E} M_{1}\left(t_{m}^{ \pm}\right)=n \mathbb{E} D_{v}\left(t_{m}^{ \pm}\right)=2 m \pm \sqrt{\omega n}
$$


Using Chebyshev's inequality and (3.17)-(3.18) it follows that, whp, $M_{1}\left(t_{m}^{-}\right)<2 m$ and $M_{1}\left(t_{m}^{+}\right)>2 m$. Since $M_{1}\left(\tau_{2 m}\right)=2 m$ by (3.10) -(3.11), using time-monotonicity of $M_{1}(t)$ this implies that, whp,

$$
t_{m}^{-}<\tau_{2 m}<t_{m}^{+}
$$

Next we focus on $\mu_{k}(\mathbf{d})=\sum_{v \in[n]} D_{v}\left(\tau_{2 m}\right)^{k} / n=M_{k}\left(\tau_{2 m}\right) / n$, see (2.5) and (3.10)-(3.11). By combining (3.8) and (3.14) with (3.15) it follows that, for every fixed integer $q \geqslant 0$,

$$
\mathbb{E}\left\langle D_{v}\left(t_{m}^{ \pm}\right)\right\rangle_{q}=\left(\frac{2 m \pm \sqrt{\omega n}}{2 m}\right)^{q} \mathbb{E}\left\langle D_{v}\left(t_{m}\right)\right\rangle_{q}=\mathbb{E}\left\langle D_{v}\left(t_{m}\right)\right\rangle_{q}+O(\sqrt{\omega / n}) .
$$

Using (3.16) again, we infer that $\mathbb{E} D_{v}\left(t_{m}^{ \pm}\right)^{k}=\mathbb{E} D_{v}\left(t_{m}\right)^{k}+O(\sqrt{\omega / n})$. Consequently, using Chebyshev's inequality and (3.17) again, we deduce that, whp,

$$
M_{k}\left(t_{m}^{ \pm}\right)=\mathbb{E} M_{k}\left(t_{m}^{ \pm}\right) \pm \sqrt{\omega n}=n \mathbb{E} D_{v}\left(t_{m}\right)^{k}+O(\sqrt{\omega n}),
$$

Since $\mathbb{E} D_{v}\left(t_{m}\right)^{k}=\mathbb{E} Y_{n}^{k}$ by (3.13), using time-monotonicity of $M_{k}(t)$ and (3.19) it now follows that

$$
M_{k}\left(\tau_{2 m}\right)=n \mathbb{E} Y_{n}^{k}+o_{\mathrm{p}}\left(\omega n^{1 / 2}\right),
$$

which in view of $\mu_{k}(\mathbf{d})=M_{k}\left(\tau_{2 m}\right) / n$ establishes (2.8).

Finally, we turn to $\pi_{k}(\mathbf{d})=\sum_{v \in[n]} \mathbb{1}_{\left\{D_{v}\left(\tau_{2 m}\right)=k\right\}} / n=N_{k}\left(\tau_{2 m}\right) / n$, see (2.5) and (3.10)-(3.11). Here the key observation is that (3.19), (3.20) and (3.18) together imply that, whp,

$$
\left|N_{k}\left(\tau_{2 m}\right)-N_{k}\left(t_{m}\right)\right| \leqslant\left|M_{1}\left(t_{m}^{+}\right)-M_{1}\left(t_{m}^{-}\right)\right| \leqslant\left|\mathbb{E} M_{1}\left(t_{m}^{+}\right)-\mathbb{E} M_{1}\left(t_{m}^{-}\right)\right|+2 \sqrt{\omega n}=4 \sqrt{\omega n} .
$$

Furthermore, $\mathbb{E} N_{k}\left(t_{m}\right)=n \mathbb{E} \mathbb{1}_{\left\{D_{v}\left(t_{m}\right)=k\right\}}=n \mathbb{P}\left(Y_{n}=k\right)$ by (3.13), and Var $N_{k}\left(t_{m}\right)=O(n)$ by independence of the birth processes. Hence, using (3.22) and Chebyshev's inequality it follows that, whp,

$$
\left|N_{k}\left(\tau_{2 m}\right)-n \mathbb{P}\left(Y_{n}=k\right)\right| \leqslant\left|N_{k}\left(t_{m}\right)-n \mathbb{P}\left(Y_{n}=k\right)\right|+4 \sqrt{\omega n} \leqslant 5 \sqrt{\omega n},
$$

which in view of $\pi_{k}(\mathbf{d})=N_{k}\left(\tau_{2 m}\right) / n$ establishes (2.7).

Proof of Lemma 3.1. We start with the first identity in (3.9), which is an immediate consequence of (2.4) and $(1-p x)^{-\alpha}=\sum_{r \in \mathbb{N}}\left(\begin{array}{c}\alpha+r-1 \\ r\end{array}\right) p^{r} x^{r}$. Differentiation then yields the second identity in (3.9), which for $x=1$ yields $\mathbb{E} Y=\alpha \cdot p /(1-p)$. Taking higher derivatives yields, for all integers $k \geqslant 1$,

$$
\mathbb{E} \prod_{0 \leqslant j<k}(Y-j)=\left(\frac{\mathrm{d}^{k}}{\mathrm{~d} p^{k}}(1-p)^{-\alpha}\right) \cdot(1-p)^{\alpha} p^{k}=\alpha(\alpha+1) \cdots(\alpha+k-1) \cdot\left(\frac{p}{1-p}\right)^{k} .
$$

Inserting $\mathbb{E} Y=\alpha \cdot p /(1-p)$ into (3.24) then readily establishes (3.8), where the case $k=0$ is trivial. Finally, (iii) follows immediately by substituting $p=1-1 /(1+x / \alpha)$ into $\mathbb{E} Y=\alpha p /(1-p)$.

Remark 3.2. We used above that the degrees can be obtained by stopping the independent processes $D_{v}(t)$ suitably. Another, related, construction that also can be used to show asymptotic results is that, for any $p \in$ $(0,1)$, if $D_{v} \sim \operatorname{NBin}(\alpha, p)$ are independent, then the degree sequence $\left(d_{v}\right)_{v \in[n]}$ has the same distribution as $\left(D_{v}\right)_{v \in[n]}$ conditioned on $\sum_{v} D_{v}=2 m$, see e.g. Holst 22] or Pittel [43, p. 624]. That the degree distribution is asymptotically negative binomial can also be shown by direct calculations [44, Case 2b, p. 153].

\subsection{Proof of Theorem 2.2; relating $G_{n, m}^{\alpha}$ with $G_{n, m}^{\alpha, *}$}

For the transfer statements of Theorem 2.2 and Remark 2.3 the strategy is to compare the stepwise conditional probabilities of the added edges in $\left(G_{n, i}^{\alpha}\right)_{i \geqslant 0}$ and its multigraph variant $\left(G_{n, i}^{\alpha, *}\right)_{i \geqslant 0}$; these probabilities are identical up to the normalizing factors in the denominator. (This is the same strategy as in [43, Section 3], although we do the details differently and somewhat simpler.) We begin by relating the two processes under an extra technical condition. 
Lemma 3.3. Define $Z\left(G_{i}\right):=\sum_{v \in[n]} d_{v}(i)^{3}$, where $d_{v}(i):=d_{v}\left(G_{i}\right)$ denotes the degree of vertex $v$ in $G_{i}$. Given $A, C, \alpha_{0}>0$, the following holds whenever $1 \leqslant m \leqslant C n$ and $\alpha \geqslant \alpha_{0}$. For any sequence $\left(G_{0}, \ldots, G_{m}\right)$ of simple graphs with $V\left(G_{i}\right)=[n], G_{i} \subset G_{i+1}, e\left(G_{i}\right)=i$, and $Z\left(G_{m-1}\right) \leqslant A n$, we have

$$
\mathbb{P}\left(\left(G_{n, i}^{\alpha}\right)_{0 \leqslant i \leqslant m}=\left(G_{0}, \ldots, G_{m}\right)\right)=\Theta(1) \cdot \mathbb{P}\left(\left(G_{n, i}^{\alpha, *}\right)_{0 \leqslant i \leqslant m}=\left(G_{0}, \ldots, G_{m}\right)\right),
$$

where the implicit constants in (3.25) may depend on $C, \alpha_{0}, A$.

Proof. Let $\{v, w\} \in\left(\begin{array}{c}{[n]} \\ 2\end{array}\right) \backslash E\left(G_{i}\right)$ denote the (unique) edge in which $G_{i+1}$ and $G_{i}$ differ. Note that, by construction, the edge $\{v, w\}$ is added to $G_{i}$ with probability proportional to $2\left(d_{v}(i)+\alpha\right)\left(d_{w}(i)+\alpha\right)$ in both random graph processes, but each time the normalizing factor in the denominator differs slightly. Indeed, recalling $\sum_{v \in[n]} d_{v}(i)=2 i$, in the random multigraph process the normalizing factor equals

$$
\begin{aligned}
\sum_{\{x, y\} \in\left(\begin{array}{c}
{\left[\begin{array}{c}
n \\
2
\end{array}\right)} \\
2
\end{array}\right.} 2\left(d_{x}(i)+\alpha\right)\left(d_{y}(i)+\alpha\right)+\sum_{x \in[n]}\left(d_{x}(i)+\alpha\right)\left(d_{y}(i)+1+\alpha\right) \\
=\left(\sum_{x \in[n]}\left(d_{x}(i)+\alpha\right)\right)^{2}+\sum_{x \in[n]}\left(d_{x}(i)+\alpha\right)=(2 i+\alpha n)(2 i+\alpha n+1),
\end{aligned}
$$

whereas in the original (simple) random graph process the normalizing factor equals

$$
\begin{aligned}
& \sum_{\{x, y\} \in\left(\begin{array}{c}
{[n]} \\
2
\end{array}\right) \backslash E\left(G_{i}\right)} 2\left(d_{x}(i)+\alpha\right)\left(d_{y}(i)+\alpha\right) \\
& =(2 i+\alpha n)^{2}-[\underbrace{2 \sum_{\{x, y\} \in E\left(G_{i}\right)}\left(d_{x}(i)+\alpha\right)\left(d_{y}(i)+\alpha\right)+\sum_{x \in[n]}\left(d_{x}(i)+\alpha\right)^{2}}_{=: Q\left(G_{i}\right)}] .
\end{aligned}
$$

Putting things together, it follows that the one-step conditional probabilities are given by

$$
\begin{aligned}
& \mathbb{P}\left(G_{n, i+1}^{\alpha}=G_{i+1} \mid\left(G_{n, j}^{\alpha}\right)_{0 \leqslant j \leqslant i}=\left(G_{0}, \ldots, G_{i}\right)\right)=\frac{2\left(d_{v}(i)+\alpha\right)\left(d_{w}(i)+\alpha\right)}{(2 i+\alpha n)^{2}-Q\left(G_{i}\right)}, \\
& \mathbb{P}\left(G_{n, i+1}^{\alpha, *}=G_{i+1} \mid\left(G_{n, j}^{\alpha, *}\right)_{0 \leqslant j \leqslant i}=\left(G_{0}, \ldots, G_{i}\right)\right)=\frac{2\left(d_{v}(i)+\alpha\right)\left(d_{w}(i)+\alpha\right)}{(2 i+\alpha n)(2 i+\alpha n+1)} .
\end{aligned}
$$

Next we claim that (3.25) follows if $\max _{0 \leqslant i<m} Q\left(G_{i}\right) \leqslant B \alpha^{2} n$ for some constant $B=B\left(C, \alpha_{0}, A\right)>0$. Indeed, the right-hand sides of (3.28) - (3.29) are then equal up to a multiplicative factor of $1+O\left(n^{-1}\right)$, where the implicit constants may depend on $B$ and $\alpha_{0}$. Noting that initially $\mathbb{P}\left(G_{n, 0}^{\alpha}=G_{0}\right)=1=\mathbb{P}\left(G_{n, 0}^{\alpha, *}=G_{0}\right)$, then (3.25) follows readily by comparing the product of $m \leqslant C n$ conditional probabilities.

It remains to prove $\max _{0 \leqslant i<m} Q\left(G_{i}\right) \leqslant B \alpha^{2} n$. Using $(x+\alpha)(y+\alpha) \leqslant 4 \max \left\{x^{2}, y^{2}, \alpha^{2}\right\}$ it follows that

$$
\begin{aligned}
Q\left(G_{i}\right) & \leqslant 8 \sum_{\{x, y\} \in E\left(G_{i}\right)}\left(d_{x}(i)^{2}+d_{y}(i)^{2}+\alpha^{2}\right)+4 \sum_{x \in[n]}\left(d_{x}(i)^{2}+\alpha^{2}\right) \\
& \leqslant 16 \sum_{v \in[n]} d_{v}(i)^{3}+8 \alpha^{2}\left|E\left(G_{i}\right)\right|+4 \sum_{v \in[n]} d_{v}(i)^{2}+4 \alpha^{2} n \leqslant 20 \cdot\left[Z\left(G_{i}\right)+\alpha^{2} \max \{i, n\}\right] .
\end{aligned}
$$

Noting that $Z\left(G_{i}\right) \leqslant Z\left(G_{m-1}\right)$ by monotonicity of the degrees, and using the assumptions $Z\left(G_{m-1}\right) \leqslant A n$, $i<m \leqslant C n$ and $\alpha \geqslant \alpha_{0}$ we readily infer $Q\left(G_{i}\right) \leqslant 20\left[A+\alpha^{2}(C+1)\right] n \leqslant B \alpha^{2} n$ for suitable $B=B\left(C, \alpha_{0}, A\right)>$ 0 , completing the proof (as discussed).

To deduce the desired transfer statements, it intuitively remains to show that typically $Z\left(G_{n, m-1}^{\alpha}\right)=O(n)$. Perhaps surprisingly, using Lemma 3.3 this can in fact be derived (or 'bootstrapped') from a corresponding bound for the more tractable process $G_{n, m}^{\alpha, *}$ by a stopping time argument. 
Proof of Theorem 2.2. Since (2.2) implies (2.1), it suffices to prove Remark 2.3, Recalling (2.5) and (2.8) with $\mathbb{E} Y_{n}^{5}=O(1)$, by Theorem 2.5 (and monotonicity in $m$ ) we may choose $A=A\left(C, \alpha_{0}\right)>0$ such that

$$
\mathbb{P}\left(Z\left(G_{n, m-1}^{\alpha, *}\right)>A n\right) \leqslant \mathbb{P}\left(Z\left(G_{n,\lfloor C n\rfloor}^{\alpha, *}\right)>A n\right)=o(1) .
$$

Next, by summing 3.25) over all graph sequences from $\mathcal{G}_{n, m}$ with $Z\left(G_{m-1}\right) \leqslant A n$ (ignoring those sequences that are not realizable by the simple random graph process $\left.\left(G_{n, i}^{\alpha}\right)_{0 \leqslant i \leqslant m}\right)$ it follows that

$$
\mathbb{P}\left(\left(G_{n, i}^{\alpha}\right)_{0 \leqslant i \leqslant m} \in \mathcal{G}_{n, m} \text { and } Z\left(G_{n, m-1}^{\alpha}\right) \leqslant A n\right) \leqslant O(1) \cdot \mathbb{P}\left(\left(G_{n, i}^{\alpha, *}\right)_{0 \leqslant i \leqslant m} \in \mathcal{G}_{n, m}\right),
$$

where here and below we use the convention that all implicit constants may depend on $C, \alpha_{0}, A$.

Finally, we compare $\mathbb{P}\left(Z\left(G_{n, m-1}^{\alpha}\right)>A n\right)$ with $\mathbb{P}\left(Z\left(G_{n, m-1}^{\alpha, *}\right)>A n\right)$. Since $Z\left(G_{i}\right)<Z\left(G_{i+1}\right)$ by monotonicity of the degrees, here the idea is to focus on the first step where $Z\left(G_{j}\right) \leqslant A n$ is violated, and then only compare the probabilities in both processes up to (and including) that step via Lemma 3.3. Turning to the details, define $\mathcal{H}_{n, j}$ as the set of all simple graph sequences $\left(G_{0}, \ldots, G_{j}\right)$ with $V\left(G_{i}\right)=[n], G_{i} \subset G_{i+1}$, $e\left(G_{i}\right)=i, Z\left(G_{j-1}\right) \leqslant A n$ and $Z\left(G_{j}\right)>A n$. Noting that initially $Z\left(G_{n, 0}^{\alpha}\right)=0=Z\left(G_{n, 0}^{\alpha, *}\right)$, by summing (3.25) over all graph sequences from $\mathcal{H}_{n, j}$ it follows that

$$
\begin{aligned}
\mathbb{P}\left(Z\left(G_{n, m-1}^{\alpha}\right)>A n\right) & =\sum_{1 \leqslant j \leqslant m-1} \mathbb{P}\left(\left(G_{n, i}^{\alpha}\right)_{0 \leqslant i \leqslant j} \in \mathcal{H}_{n, j}\right) \\
& =\Theta(1) \cdot \sum_{1 \leqslant j \leqslant m-1} \mathbb{P}\left(\left(G_{n, i}^{\alpha, *}\right)_{0 \leqslant i \leqslant j} \in \mathcal{H}_{n, j}\right) \leqslant O(1) \cdot \mathbb{P}\left(Z\left(G_{n, m-1}^{\alpha, *}\right)>A n\right),
\end{aligned}
$$

which together with (3.31)-(3.32) completes the proof of inequality (2.2).

Remark 3.4. The transfer statement of Theorem 2.2 fails when $\alpha=\alpha(n) \rightarrow 0$ sufficiently fast: e.g., for $\alpha=$ $o\left(n^{-2}\right)$ and $m=O(n)$ it is easy to check that $\left(G_{n, i}^{\alpha}\right)_{0 \leqslant i \leqslant m}$ whp sequentially builds up a clique, whereas $\left(G_{n, i}^{\alpha, *}\right)_{0 \leqslant i \leqslant m}$ whp only adds loops.

\section{Size of the giant component: proof of the main theorems}

In this section we prove our main giant component results Theorems 1.1 1.2 and 2.1 by closely following the heuristics from Section 2.2. (Recalling $\alpha=\alpha(n) \rightarrow a \in(0, \infty]$ as $n \rightarrow \infty$, we sometimes need to distinguish the cases $a<\infty$ and $a=\infty$, since they have different asymptotic degree distributions.)

Proof of Theorem 2.1. Recall that Theorem 1.1 is a special case of Theorem 1.2. Thus it suffices to prove that Theorem 1.2 holds with $L_{j}(m):=L_{j}\left(G_{n, m}^{\alpha, *}\right)$. By considering subsequences in the standard way, we may assume that $\varepsilon=\varepsilon(n) \rightarrow \varepsilon_{\infty}$, for some $\varepsilon_{\infty} \in[0, \infty)$, as $n \rightarrow \infty$. Let $Y_{n} \sim \operatorname{NBin}\left(\alpha, p_{n}\right)$ be as in Theorem 2.5], and let $\delta_{n}:=n^{-1 / 2} \omega(n)$ with $\omega(n):=\log n$, say (any sequence with $n^{-1 / 2} \ll \delta_{n} \ll n^{-1 / 3}$ would work). Then (2.7)-(2.8) show that the random vector $\delta_{n}^{-1}\left(\left(\pi_{k}(\mathbf{d})-\mathbb{P}\left(Y_{n}=k\right)\right)_{k=0}^{\infty},\left(\mu_{k}(\mathbf{d})-\mathbb{E} Y_{n}^{k}\right)_{k=0}^{\infty}\right)$ converges in probability to 0 in the product space $\mathbb{R}^{\infty} \times \mathbb{R}^{\infty}$. By the Skorohod coupling theorem [36, Theorem 4.30], we may without loss of generality assume that the random multigraphs for different $n$ are coupled such that this holds a.s., and thus that, a.s., for every for every integer $k \geqslant 0$ we have

$$
\begin{aligned}
& \pi_{k}(\mathbf{d})=\mathbb{P}\left(Y_{n}=k\right)+o\left(\delta_{n}\right), \\
& \mu_{k}(\mathbf{d})=\mathbb{E} Y_{n}^{k}+o\left(\delta_{n}\right) .
\end{aligned}
$$

As a preparatory step, we now show that $\mathbf{d}=\left(d_{v}\right)_{v \in[n]}$ satisfies the assumptions of Theorem 2.8 with

$$
D \sim \begin{cases}\operatorname{NBin}\left(a, p_{\infty}\right), & \text { if } a<\infty, \\ \operatorname{Po}\left(1+\varepsilon_{\infty}\right), & \text { if } a=\infty,\end{cases}
$$

where $p_{\infty}$ is defined as in (4.4) below. Since $m=m_{\mathrm{c}}(1+\varepsilon)$, recalling (2.6) and (1.1) we infer that, as $n \rightarrow \infty$,

$$
p_{n}=\frac{2 m_{\mathrm{c}}(1+\varepsilon)}{n \alpha+2 m_{\mathrm{c}}(1+\varepsilon)}=\frac{(1+\varepsilon) /(\alpha+1)}{1+(1+\varepsilon) /(\alpha+1)}=\frac{1+\varepsilon}{\alpha+2+\varepsilon} \rightarrow \frac{1+\varepsilon_{\infty}}{a+2+\varepsilon_{\infty}}=: p_{\infty} .
$$


If $a<\infty$, then it readily follows from (2.4) that $Y_{n} \stackrel{\mathrm{d}}{\rightarrow} \operatorname{NBin}\left(a, p_{\infty}\right)$. If instead $a=\infty$, then (4.4) yields $p_{n} \rightarrow 0$ and $\alpha p_{n} \rightarrow 1+\varepsilon_{\infty}$ as $n \rightarrow \infty$, so that (2.4) implies

$$
\mathbb{P}\left(Y_{n}=k\right)=\frac{\prod_{0 \leqslant j<k}\left(\alpha p_{n}+j p_{n}\right)}{k !}\left(1-p_{n}\right)^{\alpha} \rightarrow \frac{\left(1+\varepsilon_{\infty}\right)^{k}}{k !} e^{-\left(1+\varepsilon_{\infty}\right)}, \quad k \geqslant 0,
$$

and thus $Y_{n} \stackrel{\mathrm{d}}{\rightarrow} \operatorname{Po}\left(1+\varepsilon_{\infty}\right)$. To sum up, in both cases we have $Y_{n} \stackrel{\mathrm{d}}{\rightarrow} D$, so that (4.1) implies, a.s., $\pi_{k}(\mathbf{d})=\mathbb{P}\left(Y_{n}=k\right)+o(1) \rightarrow \mathbb{P}(D=k)$ as $n \rightarrow \infty$, for every $k \geqslant 0$. Furthermore, $\mathbb{E} D \in(0, \infty)$ and $\mathbb{P}(D=1)>0$ are obvious (note that $p_{\infty}>0$ when $a<\infty$ ). Moreover, 4.2) and Theorem 2.5 yield, a.s., $\mu_{5}(\mathbf{d})=\mathbb{E} Y_{n}^{5}+o(1)=O(1)$, so the assumptions of Theorem 2.8 hold (as claimed).

Next, gearing up to apply Theorem 2.8 in the two separate cases $\varepsilon_{\infty}=0$ and $\varepsilon_{\infty}>0$, we now estimate $\mathbb{E} D$ and $\mathbb{E} D(D-2)$. In particular, (4.3), (3.8) and (4.4) yield, when $a<\infty$,

$$
\mathbb{E} D=a \frac{p_{\infty}}{1-p_{\infty}}=\frac{a}{a+1}\left(1+\varepsilon_{\infty}\right)=\frac{1}{1+a^{-1}}\left(1+\varepsilon_{\infty}\right),
$$

which obviously holds for $a=\infty$ too (with our convention $\infty^{-1}=0$ ). Similarly, (3.8) yields, when $a<\infty$,

$$
\mathbb{E} D(D-2)=\mathbb{E} D(D-1)-\mathbb{E} D=(\mathbb{E} D)^{2}\left(1+a^{-1}\right)-\mathbb{E} D=\frac{1}{1+a^{-1}}\left(1+\varepsilon_{\infty}\right) \varepsilon_{\infty},
$$

which again holds for $a=\infty$ by standard Poisson formulas for $D \sim \operatorname{Po}\left(1+\varepsilon_{\infty}\right)$.

Case 1: $\varepsilon(n) \rightarrow \varepsilon_{\infty}>0$. In this case, (4.7) yields $\mathbb{E} D(D-2)>0$. Hence, Theorem 2.d(i) applies and shows the existence of a unique $\xi=\xi\left(a, \varepsilon_{\infty}\right) \in(0,1)$ such that

$$
\mathbb{E} D \xi^{D-1}=\xi \mathbb{E} D .
$$

Furthermore, by Theorem 2.4 and the calculations above, a.s. the degree sequence $\mathbf{d}$ is such that (2.9) applies to $G_{n, m}^{\alpha, *}$ conditioned on d. Consequently, (2.9) holds for $G_{n, m}^{\alpha, *}$ also unconditionally, which is (1.5) (1.6) with $\rho_{a}(\varepsilon)$ replaced by

$$
\rho_{a}\left(\varepsilon_{\infty}\right):=1-\mathbb{E} \xi^{D} .
$$

In the remainder we take (4.9) together with (4.4), (4.3) and (4.8) as the definition of the function $\rho_{a}\left(\varepsilon_{\infty}\right)$, for all $a \in(0, \infty]$ and $\varepsilon_{\infty} \in(0, \infty)$. We now study further properties of this function, and for convenience temporarily write $\varepsilon$ instead of $\varepsilon_{\infty}$. (We no longer consider finite $n$ here, so there is no risk of confusion.)

If $a=\infty$, then by combining $D \sim \operatorname{Po}(1+\varepsilon)$ with standard Poisson formulas, we can rewrite (4.8) as

$$
e^{-(1+\varepsilon)(1-\xi)}=\xi
$$

In view of (4.9) this also yields the identity

$$
\rho_{\infty}(\varepsilon)=1-e^{-(1+\varepsilon)(1-\xi)}=1-\xi,
$$

establishing that $\rho_{\infty}(\xi)$ is the positive solution of (1.7), as asserted.

If $a<\infty$, then by combining $D \sim \operatorname{NBin}\left(a, p_{\infty}\right)$ with (3.9) and (4.4) we can rewrite (4.8) as

$$
\left(\frac{a+1}{a+2+\varepsilon-(1+\varepsilon) \xi}\right)^{a+1}=\xi \text {. }
$$

Then (4.9) and (3.9) yield

$$
\rho_{a}(\varepsilon)=1-\left(\frac{a+1}{a+2+\varepsilon-(1+\varepsilon) \xi}\right)^{a}=1-\xi^{a /(a+1)} .
$$

By (4.13), (4.12) and elementary algebra, it also follows that

$$
\rho_{a}(\varepsilon)=1-\left(1+\frac{(1+\varepsilon)(1-\xi)}{a+1}\right) \cdot \xi=(1-\xi) \cdot\left(1-\frac{(1+\varepsilon) \xi}{a+1}\right) .
$$


We remark that, in view of (4.11), equations (4.13)-(4.14) both also hold when $a=\infty$ (by interpreting the fractions in the natural way, i.e., as limits). As a further technical interlude (that can safely be skipped on a first reading), note that the difference between the two sides in (4.12) is a convex function of $\xi \in[0,1]$, which vanishes at $\xi=\xi(a, \varepsilon)$ and $\xi=1$ but not identically. Hence its derivative at $\xi(a, \varepsilon)$ must be negative, and thus the implicit function theorem applies and shows that $\xi(a, \varepsilon)$ is an analytic function of $(a, \varepsilon) \in(0, \infty)^{2}$. Hence, using (4.13) or (4.14), it follows that $\rho_{a}(\varepsilon)$ is also an analytic function of $(a, \varepsilon)$.

Case 2: $\varepsilon(n) \rightarrow \varepsilon_{\infty}=0$. In this case, (4.7) yields $\mathbb{E} D(D-2)=0$. Using (4.6) and (3.8) when $a<\infty$ and standard Poisson formulas for $D \sim \operatorname{Po}(1)$ when $a=\infty$, it follows that $\mathbb{E} D=1 /\left(1+a^{-1}\right)$ and

$$
\frac{\mathbb{E} D}{\mathbb{E} D(D-1)(D-2)}=\frac{1}{(\mathbb{E} D)^{2}(1+1 / a)(1+2 / a)}=\frac{1+a^{-1}}{1+2 / a}
$$

Moreover, using (4.2) and $\delta_{n} \ll n^{-1 / 3} \ll \varepsilon$, in analogy with (4.6)-(4.7) it follows that, a.s.,

$$
\zeta_{n} / n=\mu_{2}(\mathbf{d})-2 \mu_{1}(\mathbf{d})=\mathbb{E} Y_{n}\left(Y_{n}-2\right)+o\left(\delta_{n}\right)=\frac{1}{1+\alpha^{-1}}(1+\varepsilon) \varepsilon+o(\varepsilon) \sim \frac{1}{1+a^{-1}} \varepsilon .
$$

Consequently, $n^{-2 / 3} \zeta_{n}=\Theta\left(\varepsilon n^{1 / 3}\right) \rightarrow \infty$, and Theorem 2.8(ii) applies a.s. to $G_{n, m}^{\alpha, *}$ conditioned on d, again using Theorem 2.4. Hence, (2.10) yields, conditioned on $\mathbf{d}$ and therefore also unconditioned,

$$
L_{1}\left(G_{n, m}^{\alpha, *}\right)=\left(\frac{2\left(1+a^{-1}\right)}{1+2 / a}+o_{\mathrm{p}}(1)\right) \zeta_{n}=\frac{2 \varepsilon n}{1+2 / a} \cdot\left(1+o_{\mathrm{p}}(1)\right) .
$$

We similarly also obtain $L_{2}\left(G_{n, m}^{\alpha, *}\right)=o_{\mathrm{p}}\left(\zeta_{n}\right)=o_{\mathrm{p}}(\varepsilon n)=o_{\mathrm{p}}\left(L_{1}\left(G_{n, m}^{\alpha, *}\right)\right)$. This verifies (1.6), in this case too. However, to derive (1.5) from (4.17), it remains to stitch the two formulas together by showing that, as $\varepsilon \rightarrow 0$ with $a \leqslant \infty$ fixed, we have $\rho_{a}(\varepsilon) \sim 2 \varepsilon /(1+2 / a)$, as asserted (more precisely) in (1.4). We shall do this by an analytic argument. (An alternative, more conceptual, proof is sketched in Remark 4.2.) Since the claimed asymptotics is well-known in the Poisson case $a=\infty$, for simplicity we henceforth assume $a<\infty$ (the proof in the simpler case $a=\infty$ proceeds in the same way). With foresight, we define

$$
\psi:=(1+\varepsilon)(1-\xi)>0,
$$

and then (similarly to the calculations leading to (4.14) above) rewrite (4.12) as

$$
\xi=\left(1+\frac{(1+\varepsilon)(1-\xi)}{a+1}\right)^{-(a+1)}=\left(1+\frac{\psi}{a+1}\right)^{-(a+1)} .
$$

Using (4.12) and $\log (1+x) \leqslant x$, it follows that

$$
(1-\xi)+(1-\xi)^{2} / 2<-\log (1-(1-\xi))=\log \frac{1}{\xi}=(a+1) \log \left(1+\frac{\psi}{a+1}\right) \leqslant \psi=(1+\varepsilon)(1-\xi),
$$

which after dividing by $1-\xi>0$ easily gives $1-\xi<2 \varepsilon$, and thus $\rho \leqslant 1-\xi<2 \varepsilon$ by (4.13) or (4.14). Furthermore, equations (4.18)-4.19) and a Taylor expansion (or the general binomial series) yield

$$
\frac{\psi}{1+\varepsilon}=1-\xi=1-\left(1+\frac{\psi}{a+1}\right)^{-(a+1)}=\psi-\frac{a+2}{2(a+1)} \psi^{2}+O\left(\psi^{3}\right) .
$$

Since $0<\psi \leqslant(1+\varepsilon) 2 \varepsilon=O(\varepsilon)$, after dividing by $\psi>0$ it then routinely follows that

$$
\psi=\frac{2(a+1) \varepsilon}{(a+2)(1+\varepsilon)}+O\left(\varepsilon^{2}\right)=\frac{2(a+1)}{a+2} \varepsilon+O\left(\varepsilon^{2}\right) .
$$

Recalling (4.18) and that $1-\xi=O(\varepsilon)$, now (4.14) and (4.22) imply for $\varepsilon \searrow 0$ the asymptotics

$$
\rho_{a}(\varepsilon)=\frac{\psi}{1+\varepsilon} \cdot\left(\frac{a}{a+1}+O(\varepsilon)\right)=\frac{2 a \varepsilon}{a+2}+O\left(\varepsilon^{2}\right),
$$

as stated in (1.4). This completes the proof of Theorem 2.1 as discussed below (4.17). 
Proof of Theorems 1.1 and 1.2. These results follow from the multigraph version Theorem 2.1] and the transfer result Theorem 2.2 by routine arguments (the key point is that any event which fails with probability at most $\pi=o(1)$ in $G_{n, m}^{\alpha, *}$ fails with probability at most $B \cdot \pi+o(1)=o(1)$ in $\left.G_{n, m}^{\alpha}\right)$.

Remark 4.1. When $\varepsilon \rightarrow \infty$ (with $\alpha$ fixed), it follows easily from (4.19) that $\xi \rightarrow 0$, and thus (4.13) implies $\rho_{a}(\varepsilon)=1-\xi^{a /(a+1)} \rightarrow 1$. When $a \rightarrow \infty$ with $\varepsilon \in(0, \infty)$ fixed, it follows easily from (4.19) that $\xi(a, \varepsilon) \rightarrow$ $\xi(\infty, \varepsilon)$ satisfying (4.10), and thus (4.14) and (4.11) imply $\rho_{a}(\varepsilon) \rightarrow 1-\xi(\infty, \varepsilon)=\rho_{\infty}(\varepsilon)$. Furthermore, since $\rho_{\infty}(\varepsilon)$ is continuous with bounded range, and each $\rho_{a}(\varepsilon)$ is increasing, it follows that $\rho_{a}(\varepsilon) \rightarrow \rho_{\infty}(\varepsilon)$ uniformly in $\varepsilon \in(0, \infty)$. We omit the details.

Remark 4.2. An alternative proof of (1.4) without calculations is based on the somewhat vague but very general idea that when we have a limit theorem with different cases as above, then the cases have to fit together smoothly. To see this in the present case, fix $\alpha$ and write for simplicity $G(n, \varepsilon):=G_{n,\left\lfloor m_{\mathrm{c}}(1+\varepsilon)\right\rfloor}^{\alpha}$. Consider a sequence $\varepsilon_{i} \rightarrow 0$. First, fix $i$ and take $\varepsilon=\varepsilon_{i}$ constant. Then, by Case $1\left(\varepsilon_{\infty}>0\right)$ in the proof above, $L_{1}\left(G\left(n, \varepsilon_{i}\right)\right)=\rho_{\alpha}\left(\varepsilon_{i}\right) n\left(1+o_{\mathrm{p}}(1)\right)$. Hence we can choose $n_{i}$ so large that with probability $>1-2^{-i}$,

$$
\left|L_{1}\left(G\left(n_{i}, \varepsilon_{i}\right)\right) / n_{i}-\rho_{\alpha}\left(\varepsilon_{i}\right)\right|<2^{-i} \varepsilon_{i} .
$$

We may further assume $n_{i+1}>n_{i}$ and $n_{i}>i \varepsilon_{i}^{-3}$. Now consider the sequence of multigraphs $G\left(n_{i}, \varepsilon_{i}\right), i \geqslant 1$. Case $2\left(\varepsilon_{\infty}=0\right)$ in the proof above applies, so (4.17) holds for the multigraphs $G\left(n_{i}, \varepsilon_{i}\right)$, which together with (4.24) implies (1.4), up to a weaker error term $o(\varepsilon)$.

It is also possible to recover the error term $O\left(\varepsilon^{2}\right)$ without explicit calculations. Suppose for definiteness that $a<\infty$. We may then solve (4.12) for $\varepsilon$, and obtain $\varepsilon=\varepsilon(\xi)$ as an analytic function of $\xi$ in some complex neighbourhood of $\xi=1$. The derivative at 1 is non-zero, e.g. as a consequence of (1.4) and (4.13), and thus the implicit function theorem shows that $\xi(a, \varepsilon)$ may be extended to an analytic function of $\varepsilon$ in some neighbourhood of 0 . By (4.13), the same holds for $\rho_{\alpha}(\varepsilon)$. Thus $\rho_{\alpha}(\varepsilon)$ is analytic on the closed half-line $[0, \infty)$, and all derivatives stay bounded as $\varepsilon \rightarrow 0$; so the error term $O\left(\varepsilon^{2}\right)$ in (1.4) follows by Taylor's theorem.

\section{$5 \quad$ Final remarks}

\subsection{Preferential attachment with negative $\alpha$}

As in previous work, we have throughout assumed $\alpha>0$. It is also possible to consider $\alpha<0$, provided $\alpha=-r$ for some integer $r=|\alpha| \in \mathbb{N}$. In this case, the process adds edges by choosing vertices as in Section 3.1 with probability proportional to $r-d_{v}$, where $d_{v}$ is the current degree. This is equivalent to starting with $r$ halfedges for each vertex, and then choosing pairs of half-edges uniformly at random, in the simple graph version conditioned on keeping the graph simple. The multigraph version can be seen (at least when $r n$ is even) as the stepwise construction of an $r$-regular multigraph by the configuration model. Equivalently, it is the process obtained if we construct an $r$-regular multigraph by the configuration model and then take its edges in uniformly random order; hence it can be seen as edge percolation on this random multigraph.

This process was introduced by Steger and Wormald [54] as a method to generate almost uniformly distributed regular graphs, see also [37, 38]. Note that no vertex will ever get degree more than $r$, so the process stops when we cannot add any edge without increasing the maximum degree above $r$. Then there are $n-O(1)$ vertices of degree $r$, in both the simple graph and multigraph version, so the final (multi)graph is almost regular. Thus, the process stops after $m=m_{\dagger}-O(1)$ steps, where

$$
m_{\dagger}:=\frac{r n}{2}=\frac{|\alpha|}{2} n .
$$

The papers [54, 37, 38] just mentioned are mainly interested in the resulting final graph, but we may also consider the entire process, and, in particular, the emergence of the giant component in this process. In the following discussion we shall always assume that $r:=|\alpha| \geqslant 3$, so that $n / 2<m_{\mathrm{c}}<n<m_{\dagger}$ by (1.1) and (5.1). Fix a small $\eta>0$ with $(1-\eta) m_{\dagger}>m_{\mathrm{c}}$. Assuming $m \leqslant(1-\eta) m_{\dagger}$, it then is fairly easy to verify that the proofs from Sections 3 4 carry over to that case (with minor routine changes). The only noteworthy 
difference is that in Section 3.2 the processes $D_{v}(t)$ are now death processes, starting with $r$ particles that each dies at rate 1 , and it turns out that (3.6) is then replaced by

$$
D_{v}(t) \sim \operatorname{Bin}\left(|\alpha|, 1-e^{-t}\right) .
$$

As a consequence, the asymptotic degree distribution is now binomial, and it follows that Theorem 2.5 holds with $Y_{n} \sim \operatorname{Bin}\left(|\alpha|, p_{n}\right)$ and $p_{n}:=2 m /(n|\alpha|)$. In spite of these differences, it turns out that the probability generating function of $Y_{n}$ can in the case $\alpha<0$ be written in the same form

$$
\mathbb{E} x^{Y_{n}}=\left(1+\frac{2 m}{n \alpha}(1-x)\right)^{-\alpha} \text {. }
$$

as in the case $\alpha>0$ (see equations (3.9) and (2.6), where $\left(1-p_{n} x\right) /\left(1-p_{n}\right)=1+p_{n}(1-x) /\left(1-p_{n}\right)$ and $p_{n} /\left(1-p_{n}\right)=2 m /(n \alpha)$ hold $)$. As a consequence, the equations 4.12) -4.14) that define $\rho_{\alpha}(\varepsilon)$ are still valid. Hence, assuming $r \geqslant 3$, Theorems 1.1, 1.2 and 2.1 hold for $\alpha:=-r$ too (the assumption $m \leqslant(1-\eta) m_{\dagger}$ may be eliminated by the same argument as in Remark 1.5). We may also let $\alpha \rightarrow-\infty$, with $\rho_{-\infty}=\rho_{\infty}$; we leave the routine details to the reader. (In the multigraph case, these results are special cases of earlier results on edge percolation in random multigraphs with given vertex degrees, see [16, 27].)

\subsection{Extensions and variants}

\subsubsection{Hypergraphs and different vertex weights}

It seems possible to adapt the methods of this paper to study hypergraph variants of the preferential attachment random graph process. Similarly, it also seems possible to analyze the natural variant where a new edge $\{v, w\}$ is added with probability proportional to $\left(d_{v}+\alpha_{v}\right)\left(d_{w}+\alpha_{w}\right)$, i.e., where each vertex has its own 'preference weight' (though here some assumptions on the $\left(\alpha_{v}\right)_{v \in[n]}$ seem necessary in order to prove a multigraph transfer statement akin to Theorem 2.2). We leave these extensions to the reader.

\subsubsection{More general preferential attachment functions}

As suggested by Lovász, see [43], and partly already by Erdős and Rényi [15], it is natural to study processes with more general preferential attachment functions $f=f_{n}: \mathbb{N} \rightarrow[0, \infty)$. We write $\left(G_{n, m}^{f}\right)_{m \geqslant 0}$ for the variant of $\left(G_{n, m}^{\alpha}\right)_{m \geqslant 0}$ where the next edge connects two currently non-adjacent $v$ and $w$ with probability proportional to $f\left(d_{v}\right) f\left(d_{w}\right)$. Similarly, we write $\left(G_{n, m}^{f, *}\right)_{m \geqslant 0}$ for the multigraph variant where the next edge connects distinct $v$ and $w$ with probability proportional to $2 f\left(d_{v}\right) f\left(d_{w}\right)$, and forms a loop at $v$ with probability proportional to $f\left(d_{v}\right) f\left(d_{v}+1\right)$. This general class of dynamic network models not only contains the preferential attachment process (via $f(k):=k+\alpha)$ but also the classical Erdős-Rényi process $($ via $f(k):=1)$, the configuration model for $d$-regular graphs and the Steger-Wormald process [54] discussed in Section [5.1] (via $f(k):=\max \{d-k, 0\}$ ), and the random $d$-process [51, 57] (via $f(k):=\mathbb{1}_{\{k<d\}}$ ).

Here the problem of determining the asymptotic degree distribution after $m$ steps is feasible for many functions $f$ via the pure birth-process based construction of $\left(G_{n, m}^{f, *}\right)_{m \geqslant 0}$ from Section [3.2, with birthrates $\lambda_{k}:=f(k)$. For example, using this construction they are easily seen to be asymptotically Poisson in the Erdős-Rényi process, and truncated Poisson in the random $d$-process. By contrast, the giant component problem for general functions $f$ appears to be more challenging. The crux is that the conditional equivalence argument from Section 3.1 (which crucially allowed us to work with the configuration model) seemingly only carries over to linear functions, which motivates the following conceptually interesting problem.

Problem 5.1. Study the giant component problem for $G_{n, m}^{f}$ or $G_{n, m}^{f, *}$ when $f$ is non-linear.

\subsubsection{Edge-rewiring variant without edge-growth}

There is a natural variant of the preferential attachment random multigraph process where the number of edges $m=\Theta(n)$ is also fixed; this was proposed in the complex networks literature by Dorogovtsev, Mendes and Samukhin around 2002 (see [12, Section 3] and [11, Chapter 4.3]). To be more precise, this preferential attachment edge-rewiring process starts with a given (arbitrary) initial multigraph $G_{0}$ with vertex set $[n]$ and $m$ multiedges, and then proceeds stepwise as follows: a uniform endvertex $v$ of a uniformly chosen edge $e$ 
is selected, and then $e$ is replaced with the edge $\{v, w\}$, where $w$ is chosen with probability proportional to $d_{w}+\alpha$. This rewiring process (also called simple edge-selection process 23] or edge reconnecting model [45]) converges rapidly to a unique stationary distribution $G_{n, m}^{\alpha, \infty}$ (see [23, Sections 1.1-1.2], [45, Section 2.2], and the 'equilibrium' discussion in [12, 11]), which in fact has the same distribution as $G_{n, m}^{\alpha, *}$ (see [45, Lemma 2.1]). It follows that we can use the random multigraph $G_{n, m}^{\alpha, *}$ to derive the long-run asymptotic properties of the edge-rewiring process with $n$ vertices and $m$ edges. In particular, the result by Pittel [43] (or our Theorem 1.1 and 2.1) confirms Conjecture 4.3 of Hruz and Peter 23] regarding the existence of a giant component.

\subsection{Other properties}

The method used in this paper, where the auxiliary results from Section 2 are combined with results for $G_{\mathbf{d}}^{*}$, can also be used to study other properties of of $G_{n, m}^{\alpha}$ and $G_{n, m}^{\alpha, *}$. We briefly mention a few selected examples of interest here (leaving details to the reader).

First, we consider the number of vertices in 'small' components of size $k=O(1)$. Under the assumptions of Theorem 2.8, in $G_{\mathbf{d}}^{*}$ their typical number can easily be related via [28, Lemma 4.1] to a branching process $\mathfrak{X}$ that depends on (a size-biased version of) the 'idealized degree distribution' $D$ from Theorem 2.5] A routine analysis of $\mathbb{P}(|\mathfrak{X}|=k)$ then leads to a conceptually simple proof of the counting formula [43, Lemma 4(b)] 8

Secondly, the so-called susceptibility, which is the expected component-size of a randomly chosen vertex, can be similarly estimated via $\mathbb{E}|\mathfrak{X}|$ in the 'subcritical' case, see [28, Corollary 3.2] and Appendix A.1.

Thirdly, the widely studied $k$-core is the largest induced subgraph with minimum vertex degree at least $k$ (which can be empty). By mimicking the first part of Theorem 2.1] one can use [31, Theorem 2.3] with $D$ from Theorem 2.5 to show that, for each $k \geqslant 2$, a linear-sized $k$-core whp appears in $G_{n, m}^{\alpha}$ and $G_{n, m}^{\alpha, *}$ around $c_{k} n$ steps, where $c_{k}:=\frac{1}{2} \inf _{\mu>0} \mu / \mathbb{P}\left(Z_{\alpha}(\mu) \geqslant k-1\right)$ with negative binomial random variable $Z_{\alpha}(\mu) \sim$ $\operatorname{NBin}(\alpha+1, \mu /(\alpha+\mu))$.

Finally, we emphasize that our methods are geared towards the spare case $m=O(n)$. Indeed, for denser graphs the reduction of the simple graph process to the multigraph variant breaks down, at least in the present form (see Theorem 2.2). This limitation is not just a mere proof artifact: in the multigraph process the threshold for connectivity is located around $n^{1+\alpha^{-1}}$ edges [43, Theorem 2], so for $\alpha<1$ it must differ from the connectivity threshold of the simple graph process (whose location remains an open problem).

\subsection{Further open problems}

We close with some open problems for the preferential attachment process $\left(G_{n, m}^{\alpha}\right)_{m \geqslant 0}$ phase transition, which are all inspired by the corresponding behaviour of the Erdős-Rényi reference model:

Problem 5.2. In the subcritical phase $m=m_{\mathrm{c}}(1-\varepsilon)$ with $\varepsilon=o(1)$ and $\varepsilon^{3} n \rightarrow \infty$, show that whp $L_{1}\left(m_{\mathrm{c}}(1-\right.$ $\varepsilon)) \sim C_{\alpha} \varepsilon^{-2} \log \left(\varepsilon^{3} n\right)$ for some constant $C_{\alpha}>0$ (sharpening [43]), and prove a variant of Corollary [1.3.

Problem 5.3. In the critical window $m=m_{\mathrm{c}}(1+\varepsilon)$ with $|\varepsilon|=O\left(n^{-1 / 3}\right)$, writing $L_{j}=L_{j}\left(G_{n, m}^{\alpha}\right)$ for the size of the $j$ th largest component of $G_{n, m}^{\alpha}$, show that the sequence $\left(L_{j} / n^{2 / 3}\right)_{j \geqslant 1}$ converges to a limiting distribution. (43] establishes that $L_{j}$ is of order $n^{2 / 3}$, for any fixed $j \geqslant 1$.) For the random multigraph $G_{n, m}^{\alpha, *}$ this can be shown by combining our conditioning approach with the configuration model results from [10], but it remains open for $G_{n, m}^{\alpha}$ (as Theorem 2.2 does not permit transfer of convergence in distribution). Also, does the resulting limiting distribution equal the corresponding Erdös-Rényi one when $\alpha=\omega\left(n^{2 / 3}\right)$ ?

Problem 5.4. In the supercritical phase $m=m_{\mathrm{c}}(1+\varepsilon)$ with $\varepsilon=o(1)$ and $\varepsilon^{3} n \rightarrow \infty$, show that (1.6) can be improved to whp $L_{2}\left(m_{\mathrm{c}}(1+\varepsilon)\right) \leqslant D_{\alpha} \varepsilon^{-2} \log \left(\varepsilon^{3} n\right)$ for some constant $D_{\alpha}>0$.

Acknowledgements. Part of this work was carried out during the authors' visit to the Isaac Newton Institute for Mathematical Sciences during the programme Theoretical Foundations for Statistical Network Analysis (EPSCR Grant Number EP/K032208/1).

\footnotetext{
${ }^{8}$ Pittel's result [43, Lemma 4(b)] is for the expected number of tree-components, and allows for growing $k$.
} 


\section{References}

[1] K.B. Athreya and P.E. Ney. Branching Processes. Springer-Verlag, Berlin (1972)

[2] A.-L. Barabási. Network Science. Cambridge University Press (2016).

[3] A.-L. Barabási and R. Albert. Emergence of scaling in random networks. Science 286 (1999), 509-512.

[4] E. Ben-Naim and P.L. Krapivsky. Popularity-driven networking. EPL (Europhysics Letters) 97 (2012), 48003.

[5] B. Bollobás. Random Graphs. 2nd ed., Cambridge University Press (2001).

[6] B. Bollobás and O. Riordan. Random graphs and branching processes. In Handbook of Large-Scale Random Networks, Bolyai Soc. Math. Stud 18 (2009), pp. 15-115.

[7] B. Bollobás and O. Riordan. The phase transition in the Erdős-Rényi random graph process. In Erdös Centennial, Bolyai Soc. Math. Stud 25 (2013), pp. 59-110.

[8] B. Bollobás, O. Riordan, J. Spencer, and G. Tusnády. The degree sequence of a scale-free random graph process. Rand. Struct. ES Algor. 18 (2001), 279-290.

[9] C. Borgs, J. Chayes, L. Lovász, V. Sós, and K. Vesztergombi. Limits of randomly grown graph sequences. European J. Combin. 32 (2011), 985-999.

[10] S. Dhara, R. van der Hofstad, J.S.H. van Leeuwaarden and S. Sen. Critical window for the configuration model: finite third moment degrees. Electron. J. Probab. 22 (2017), Paper 16, 33 pp.

[11] S.N. Dorogovtsev and J.F.F. Mendes. Evolution of Networks. Oxford University Press (2003).

[12] S.N. Dorogovtsev, J.F.F. Mendes, and A.N. Samukhin. Principles of statistical mechanics of uncorrelated random networks. Nuclear Phys. B 666 (2003), 396-416.

[13] R. Durrett. Random Graph Dynamics. Cambridge University Press (2010).

[14] P. Erdős and A. Rényi. On the evolution of random graphs. Magyar Tud. Akad. Mat. Kutató Int. Közl 5 (1960), $17-61$.

[15] P. Erdős and A. Rényi. On the evolution of random graphs. Bull. Inst. Internat. Statist., 38 (1961), 343-347.

[16] N. Fountoulakis. Percolation on sparse random graphs with given degree sequence. Internet Math. 4 (2007), 329-356.

[17] A. Frieze and M. Karoński. Introduction to Random Graphs. Cambridge University Press (2016).

[18] G. Grimmett and D. Stirzaker. Probability and Random Processes. 3rd ed., Oxford University Press (2001).

[19] R. van der Hofstad. Random Graphs and Complex Networks: Vol. 1. Cambridge University Press (2017).

[20] R. van der Hofstad, S. Janson and M. Luczak. Component structure of the configuration model: barely supercritical case. Rand. Struct. \&6 Algor., Early View (2019).

[21] R. van der Hofstad and A. Nachmias. Hypercube percolation. J. Eur. Math. Soc. 19 (2017), 725-814.

[22] L. Holst. A unified approach to limit theorems for urn models. J. Appl. Probab. 16 (1979), 154-162.

[23] T. Hruz and U. Peter. Nongrowing preferential attachment random graphs. Internet Math. 6 (2010), 461-487.

[24] T. Hulshof and A. Nachmias. Slightly subcritical hypercube percolation. Preprint (2016). arXiv: 1612.01772.

[25] S. Janson. Functional limit theorems for multitype branching processes and generalized Pólya urns. Stochastic Process. Appl. 110 (2004), 177-245.

[26] S. Janson. Limit theorems for triangular urn schemes. Probab. Theory Related Fields 134 (2006), 417-452.

[27] S. Janson. On percolation in random graphs with given vertex degrees. Electron. J. Probab. 14 (2009), 87-118.

[28] S. Janson. Susceptibility of random graphs with given vertex degrees. J. Comb. 1 (2010), 357-387.

[29] S. Janson. Probability asymptotics: notes on notation. Preprint (2011). arXiv:1108.3924

[30] S. Janson. On edge exchangeable random graphs. J. Stat. Phys. 173 (2018), 448-484.

[31] S. Janson and M. Luczak. A simple solution to the $k$-core problem. Rand. Struct. ES Algor. 30 (2007), 50-62.

[32] S. Janson and M. Luczak. A new approach to the giant component problem. Rand. Struct. ES Algor. 34 (2009), 197-216.

[33] S. Janson, T. Łuczak and A. Ruciński. Random Graphs. Wiley-Interscience (2000).

[34] S. Janson and L. Warnke. On the critical probability in percolation. Electron. J. Probab. 23 (2018), Paper 1.

[35] N.L. Johnson and S. Kotz. Urn Models and Their Applications. Wiley, New York (1977).

[36] O. Kallenberg. Foundations of Modern Probability. 2nd ed., Springer, New York (2002).

[37] J.H. Kim and V.H. Vu. Sandwiching random graphs: universality between random graph models. Adv. Math. 188 (2004), 444-469.

[38] J.H. Kim and V.H. Vu. Generating random regular graphs. Combinatorica, 26 (2006), 683-708.

[39] P.L. Krapivsky, S. Redner, and E. Ben-Naim. A Kinetic View of Statistical Physics. Cambridge University Press (2010).

[40] A.A. Markov. Sur quelques formules limites du calcul des probabilités. (Russian.) Bulletin de l'Académie Impériale des Sciences 11 (1917), 177-186.

[41] M. Molloy and B. Reed. The size of the giant component of a random graph with a given degree sequence. Combin. Probab. Comput. 7 (1998), 295-305. 
[42] A. Nachmias and Y. Peres. Critical percolation on random regular graphs. Rand. Struct. E6 Algor. 36 (2010), 111-148.

[43] B. Pittel. On a random graph evolving by degrees. Adv. Math 223 (2010), 619-671.

[44] G. Pólya. Sur quelques points de la théorie des probabilités. Ann. Inst. H. Poincaré 1 (1930), $117-161$.

[45] B. Ráth and L. Szakács. Multigraph limit of the dense configuration model and the preferential attachment graph. Acta Math. Hungar. 136 (2012), 196-221.

[46] O. Riordan. The phase transition in the configuration model. Combin. Probab. Comput. 21 (2012), 265-299.

[47] O. Riordan and L. Warnke. Explosive percolation is continuous. Science 333 (2011), 322-324.

[48] O. Riordan and L. Warnke. Achlioptas process phase transitions are continuous. Ann. Appl. Probab. 22 (2012), 1450-1464.

[49] O. Riordan and L. Warnke. The evolution of subcritical Achlioptas processes. Rand. Struct. E Algor. 47 (2015), $174-203$.

[50] O. Riordan and L. Warnke. The phase transition in bounded-size Achlioptas processes. Preprint (2017). arXiv: 1704.08714.

[51] A. Ruciński and N.C. Wormald. Random graph processes with degree restrictions. Combin. Probab. Comput. 1 (1992), 169-180.

[52] V.K. Samalam. Preferential attachment alone is not sufficient to generate scale free random networks. Preprint (2012). arXiv:1202.1498.

[53] J. Spencer and N.C. Wormald. Birth control for giants. Combinatorica 27 (2007), 587-628.

[54] A. Steger and N.C. Wormald. Generating random regular graphs quickly. Combin. Probab. Comput. 8 (1999), 377-396.

[55] S. Tavaré. The genealogy of the birth, death, and immigration process. In Mathematical Evolutionary Theory, Princeton University Press (1989), pp. 41-56.

[56] L. Warnke. On Wormald's differential equation method. Manuscript (2019).

[57] L. Warnke and N. Wormald. In preparation (2019).

[58] N.C. Wormald. Differential equations for random processes and random graphs. Ann. Appl. Probab. 5 (1995), $1217-1235$.

[59] N.C. Wormald. The differential equation method for random graph processes and greedy algorithms. In Lectures on approximation and randomized algorithms, pp. 73-155. PWN, Warsaw (1999).

\section{A Appendix}

\section{A.1 Heuristic for the phase transition location $m_{\mathrm{c}}$}

In this appendix we give an informal explanation for why $m_{\mathrm{c}}=\frac{n}{2\left(1+\alpha^{-1}\right)}$ should be the phase transition location, based on the widely-used heuristic [53, 39, 49, 34, 57] which predicts $m_{\mathrm{c}}$ as the blow-up point of the susceptibility $S(m)=S\left(G_{n, m}^{\alpha}\right):=\sum_{j}\left|C_{j}\right|^{2} / n$, where $C_{1}, C_{2}, \ldots$ denote the components of $G_{n, m}^{\alpha}$. For simplicity, we henceforth assume that there is a deterministic approximation

$$
S(m) \approx s(t) \text { with } \quad t=t(m):=m / n
$$

By equation (1.11) in Section 1.2 any two distinct tree components $C_{j}, C_{k}$ merge with probability proportional to $\left[(2+\alpha)\left|C_{j}\right|-2\right] \cdot\left[(2+\alpha)\left|C_{k}\right|-2\right]$, in which case the susceptibility changes by $\left(\left|C_{j}\right|+\left|C_{k}\right|\right)^{2} / n-\left(\left|C_{j}\right|^{2}+\right.$ $\left.\left|C_{k}\right|^{2}\right) / n=2\left|C_{j}\right|\left|C_{k}\right| / n$. Pretending that nearly all components are not-too-big tree-components (which in analogy with the Erdős-Rényi process seems reasonable, since here we are only interested in steps leading up to the critical point, i.e., before the giant emerges), with some hand-waving we loosely expect that

$$
\begin{aligned}
\mathbb{E}(S(m+1)-S(m) \mid S(m)) & \approx \sum_{j \neq k}\left(\frac{\left[(2+\alpha)\left|C_{j}\right|-2\right] \cdot\left[(2+\alpha)\left|C_{k}\right|-2\right]}{\sum_{v \neq w: \text { non-adj. }}\left(d_{v}+\alpha\right)\left(d_{w}+\alpha\right)} \cdot \frac{2\left|C_{j}\right|\left|C_{k}\right|}{n}\right) \\
& \approx \frac{2}{n} \cdot \frac{\left((2+\alpha) \sum_{j}\left|C_{j}\right|^{2}-2 \sum_{j}\left|C_{j}\right|\right)^{2}}{\left(\sum_{v}\left(d_{v}+\alpha\right)\right)^{2}}=\frac{2}{n}\left(\frac{(2+\alpha) S(m)-2}{2 i / n+\alpha}\right)^{2} .
\end{aligned}
$$

Inserting (A.1), this suggests (together with $S(m+1)-S(m) \approx s^{\prime}(t) / n$ and $S(0)=1$ ) the differential equation

$$
s^{\prime}(t)=2\left(\frac{(2+\alpha) s(t)-2}{2 t+\alpha}\right)^{2} \quad \text { and } \quad s(0)=1 \text {. }
$$


The solution $s(t)=\frac{\alpha-2 t}{\alpha-2(\alpha+1) t}$ blows up at time $t_{\mathrm{c}}:=\frac{1}{2\left(1+\alpha^{-1}\right)}$, so the described heuristic indeed predicts the critical point $m_{\mathrm{c}}=t_{\mathrm{c}} n$. (For comparison, in the Erdös-Rényi process the critical point is $n / 2$ and its susceptibility blows up at time $1 / 2$, since we analogously arrive at $s^{\prime}(t)=2 s(t)^{2}$ and $s(t)=1 /(1-2 t)$.)

One of the arguments in [4] is essentially equivalent to this heuristic (using a different time scale, see Section A.2.2. The assumed approximation (A.1) can be justified rigorously, e.g., using either the differential equation method [58, 59, 56], or the configuration model transfer method from Section 5.3.

\section{A.2 Compatibility with previous work}

In this appendix we show that our giant component results are compatible with previous work [43, 4].

\section{A.2.1 Work of Pittel (On a random graph evolving by degrees)}

Translating to our notation, Pittel [43] considers the preferential attachment random graph process for constant $\alpha \in(0, \infty)$. For $c>c_{\alpha}:=1 /\left(1+\alpha^{-1}\right)$ he defines $c^{*}$ as the (unique) root $x \in\left(0, c_{\alpha}\right)$ of

$$
\frac{x \alpha^{\alpha+1}}{(\alpha+x)^{\alpha+2}}=\frac{c \alpha^{\alpha+1}}{(\alpha+c)^{\alpha+2}} .
$$

Assuming $n^{1 / 4}\left(c-c_{\alpha}\right) \rightarrow \infty$, then [43, Theorem 1] ensures that the largest component has size

$$
L_{1}(c n / 2)=\left[1-\left(\frac{\alpha+c^{*}}{\alpha+c}\right)^{\alpha}\right] n \cdot\left(1+o_{p}(1)\right) .
$$

Using the following auxiliary claim, Theorem 1.1 implies (A.5) under the weaker assumption $n^{1 / 3}\left(c-c_{\alpha}\right) \rightarrow \infty$ conjectured in [43] (since for $m:=m_{\mathrm{c}}(1+\varepsilon)$ we have $c=(1+\varepsilon) c_{\alpha}$ and $n^{1 / 3} \varepsilon=\Theta\left(n^{1 / 3}\left(c-c_{\alpha}\right)\right) \rightarrow \infty$ ).

Claim A.1. Let $\varepsilon>0$. Then for $c:=(1+\varepsilon) c_{\alpha}$ we have $\rho_{\alpha}(\varepsilon)=1-\left(\frac{\alpha+c^{*}}{\alpha+c}\right)^{\alpha}$.

Proof. In view of (A.4) let

$$
\xi:=\frac{c^{*}(\alpha+c)}{c\left(\alpha+c^{*}\right)}=\left(\frac{\alpha+c^{*}}{\alpha+c}\right)^{\alpha+1},
$$

and observe that $\xi \in(0,1)$ holds (since $\alpha \in(0, \infty)$ and $\left.c^{*}<c_{\alpha}<c\right)$. Noting that

$$
1-\xi=\frac{c\left(\alpha+c^{*}\right)-c^{*}(\alpha+c)}{c\left(\alpha+c^{*}\right)}=\frac{\alpha\left(c-c^{*}\right)}{c\left(\alpha+c^{*}\right)},
$$

we infer

$$
\frac{\alpha+c}{\alpha+c^{*}}=1+\frac{c-c^{*}}{\alpha+c^{*}}=1+\frac{c}{\alpha} \cdot(1-\xi)=1+\frac{1+\varepsilon}{\alpha+1}(1-\xi) . .
$$

Inserting this back into (A.6), a comparison with the first equation in (4.19) now shows that $\xi$ is indeed the unique solution in $(0,1)$ to $\mathbb{E} D \xi^{D-1}=\xi \mathbb{E} D$, and thus, by (4.13) and (A.6), we obtain $\rho_{\alpha}(\varepsilon)=1-\xi^{\alpha /(\alpha+1)}=$ $1-\left(\frac{\alpha+c^{*}}{\alpha+c}\right)^{\alpha}$.

\section{A.2.2 Work of Ben-Naim and Krapivsky (Popularity-driven networking)}

Ben-Naim and Krapivsky [4] investigate the special case $\alpha=1$ of the preferential attachment random multigraph process (using a different time-parametrization) via the kinetic theory methodology from statistical physics, also called rate equation approach. Inspecting equation (7) in [4] (solving $2 m / n=\langle j\rangle=t /(1-t)$ for $t$ ), for fixed $\varepsilon>0$ it follows that adding $m=(1+\varepsilon) n / 4$ edges corresponds to their time

$$
t:=(1+n / 2 m)^{-1}=1 / 3+2 \varepsilon / 9+O\left(\varepsilon^{2}\right) .
$$

The discussion of the function $g(t)$ in 4 , p. 4, together with (1)] then says that the asymptotic fraction of vertices in the giant component should be approximately equal to

$$
3(t-1 / 3) \sim 2 \varepsilon / 3 \sim \rho_{1}(\varepsilon) \quad \text { as } \varepsilon \searrow 0,
$$

which is made rigorous by Theorems 1.1 and 2.1. Finally, noting that (A.9) and (2.6) imply the identity $p_{n}=t$ (when $\alpha=1$ ), Theorem 2.5 also gives a rigorous version of the asymptotic degree distribution (9) in [4]. 